\title{
CSF MicroRNAs Reveal Impairment of Angiogenesis and Autophagy in Parkinson Disease
}

Alan J. Fowler, MS, * Jaeil Ahn, PhD,* Michaeline Hebron, MS, Timothy Chiu, BS, Reem Ayoub, BS, Sanjana Mulki, MS, Habtom Ressom, PhD, Yasar Torres-Yaghi, MD, Barbara Wilmarth, NP, Fernando L. Pagan, MD, and Charbel Moussa, MBBS, PhD

Neurol Genet 2021;7:e633. doi:10.1212/NXG.0000000000000633

\section{Abstract}

\section{Background and Objectives}

We assessed longitudinal changes in CSF microRNAs (miRNAs) in patients with moderately severe Parkinson disease.

\section{Methods}

We used next-generation whole-genome miRNA sequencing to determine CSF miRNA expression in 75 patients with Parkinson disease after single random ascending doses of nilotinib and longitudinal miRNA expression after daily nilotinib, 150 and $300 \mathrm{mg}$, vs placebo for 1 year.

\section{Results}

Significant changes in the expression of miRNAs that control genes and pathways that regulate angiogenesis, autophagy, and the blood-brain-barrier components, primarily collagen, were observed over 1 year, suggesting impairment of these pathways in Parkinson progression in these patients. Different miRNAs that indicate activation of genes associated with autophagy flux and clearance and angiogenesis were significantly altered in the nilotinib, $300 \mathrm{mg}$ vs $150 \mathrm{mg}$, or placebo group, and these changes correlated with clinical outcomes. No changes were observed in miRNAs after a single dose of nilotinib vs placebo.

\section{Discussion}

This study suggests vascular and autophagy defects in Parkinson progression. Nilotinib, $300 \mathrm{mg}$, reverses these effects via alteration of miRNA expression, suggesting epigenomic changes that may underlie long-term disease-modifying effects.

\section{Trial Registration Information}

Clinical trial registration number: NCT02954978.
Correspondence

Dr. Moussa

cem46@georgetown.edu 


\section{Glossary}

$\mathbf{B B B}=$ blood-brain barrier; $\mathbf{D D R}=$ discoidin domain receptor; $\mathbf{D E M}=$ differentially expressed miRNA; $\mathbf{E C M}=$ extracellular matrix; FC = fold change; FR = fold regulation; GO = gene ontology; IC50 = half-maximal inhibitor concentration; LAMP = lysosome-associated membrane protein; MDS-UPDRS = Movement Disorders Society-Unified Parkinson's Disease Rating Scale; ME-ANOVA = mixed-effects analysis of variance; miRNA = microRNA; MMP = matrix metalloprotease; $\mathbf{M O C A}=$ Montreal Cognitive Assessment; TKI = tyrosine kinase inhibition; UMI = Unique Molecular Identifier; UPDRS = Unified Parkinson's Disease Rating Scale; PD = Parkinson disease; PDQ39 = Parkinson's Disease Questionnaire 39; VEGF = vascular endothelial growth factor.

MicroRNAs (miRNAs) are posttranscriptional regulators of gene expression, which predominantly act by silencing target mRNA. A number of miRNAs that control genes associated with ubiquitination and autophagy were identified in the CSF of patients, ${ }^{1}$ and these miRNAs were altered in congruence with the impairment of autophagy in postmortem human brains. ${ }^{2,3}$ Two isoforms of the tyrosine kinase discoidin domain receptors (DDRs), DDR1 and DDR2, are upregulated in the nigrostriatum of postmortem Parkinson disease (PD). ${ }^{4}$ DDR1 activation via collagen ${ }^{5}$ alters microglial activity and matrix metalloproteases (MMPs), leading to blood-brainbarrier (BBB) degradation. ${ }^{6}$ Deletion or inhibition of DDR1 attenuates neuroinflammation and improves CNS autophagy and vesicular transport. ${ }^{1,4,7-9}$

Nilotinib is a tyrosine kinase inhibitor that attenuates or reverses the level of CSF miRNAs, which regulate autophagy in PD, ${ }^{1}$ in agreement with the preclinical effects of nilotinib on molecular pathways of autophagy. ${ }^{9-16}$ Nilotinib inhibits DDR1 with a halfmaximal inhibitory concentration $\left(\mathrm{IC}_{50}\right)$ of $1 \mathrm{nM}^{7,17-20}$ Nilotinib is FDA approved for the treatment of chronic myelogenous leukemia because it inhibits $\left(\mathrm{IC}_{50}>20 \mathrm{nM}\right)$ Abelson TK. Importantly, treatment with nilotinib, 150 or $300 \mathrm{mg}$, results in a maximum CSF concentration $\left(\mathrm{C}_{\max }\right)$ of 1.9 and $4.12 \mathrm{nM}$, respectively, in patients with $\mathrm{PD},{ }^{14-16}$ therefore achieving a pharmacologically adequate concentration that would inhibit DDR1. Here, we observe significant alterations in autophagy and angiogenesis miRNAs in PD progression, but nilotinib, 300 vs $150 \mathrm{mg}$, or placebo, reverses these miRNA changes.

\section{Methods}

\section{Study Design}

The primary research objective of this study was to assess alterations in CSF microRNAs in individuals with moderately severe PD stabilized on the standard of care for dopamine replacement therapies as previously indicated. ${ }^{15,16,21}$ Participants with PD $(\mathrm{n}=75)$ were randomized to receive a single dose of placebo, $150 \mathrm{mg}, 200 \mathrm{mg}, 300$, or $400 \mathrm{mg}$ nilotinib $(\mathrm{n}=15)$ in a single-ascending dose study. ${ }^{16} \mathrm{CSF}$ was collected between 1 and 4 hours after administration of nilotinib. All participants $(\mathrm{n}=75)$ were rerandomized to receive placebo $(\mathrm{n}=21), 150 \mathrm{mg}(\mathrm{n}=$ $21)$, or $300 \mathrm{mg}$ nilotinib $(\mathrm{n}=20)$ daily for 12 months in a longitudinal study, and CSF was collected at the 12-month study visit. ${ }^{15}$ After a 3 -month washout period, eligible participants $(\mathrm{n}=$
63) were rerandomized to receive 150 or $300 \mathrm{mg}$ nilotinib for 12 months in an open-label extension phase of the study. ${ }^{21}$

\section{Study Approval}

The clinical trial (NCT02954978) was previously described and approved by the Georgetown-Howard Universities Center for clinical and Translational Science and the Georgetown University Medical Center Institutional Review Board under protocol \#2014-0006. ${ }^{15,16,21}$

An independent data safety and monitoring board that included a cardiologist, hematologist, clinical pharmacologist, neurologist, and biostatistician monitored patients' safety in the study. All samples collected and used for experiments presented in this study were deidentified and relabeled with a study ID.

\section{Participants and Clinical Outcomes}

PD diagnosis was confirmed according to the UK Brain Bank diagnostic criteria, with Hoehn and Yahr stage 2.5-3.0, Montreal Cognitive Assessment (MoCA) score 22 or higher, and Movement Disorders Society-Unified Parkinson's Disease Rating Scale (MDS-UPDRS)-III motor score 20 - 40. All participants provided written informed consent, and their treatment was optimized with current PD medications, including levodopa, $800 \mathrm{mg} / \mathrm{d}$, or less, and levodopa and/or dopamine agonists approximately 1-2 months before consenting and undergoing screening. MoCA and MDS-UPDRS, Parkinson Disease Questionnaire (PDQ39) were collected at baseline, 12-month, and 27-month study visits.

\section{CSF Collection}

CSF was collected via lumbar puncture, which was performed on all patients between 1 and 4 hours after the first dose of nilotinib and at 12 months treated. The first $1 \mathrm{~mL}$ of CSF collected was discarded, and all samples were centrifuged at $1000 \times \mathrm{g}$ for 15 minutes before being aliquoted and stored at $-80^{\circ} \mathrm{C}$. Human hemoglobin levels were measured (Bethyl Lab Inc., E30-136), and samples containing $>25 \mathrm{ng} / \mathrm{mL}$ hemoglobin were eliminated from the study.

\section{MicroRNA Sequencing}

MicroRNA sequencing was conducted as previously described. ${ }^{1}$ Briefly, cell-free total RNA was isolated from $200 \mu \mathrm{L}$ of CSF using the Qiagen miRNAeasy serum/plasma extraction kit (Qiagen, 217184). Cursory Quality control analysis to confirm RNA was performed on each sample using UV-VIS spectroscopy on a Nanodrop ND-1000 (ThermoFisher). As 
per the manufacturer's instructions, samples were normalized to an input volume of $5 \mu \mathrm{L}$ RNA eluate to prepare miRNAseq libraries using the Qiagen QiaSeq miRNA-seq library preparation kit (Qiagen, 331502). Next-generation sequencing was performed on a NextSeq 550 Sequencing System (Illumina) using single-end (SE) $1 \times 75$ base pairs sequencing to a depth of 25 million raw reads per sample. FASTQ files are uploaded to the online Qiagen Data Analysis Center for miRNA quantification. In the primary QIAseq quantification step, the Unique Molecular Identifier (UMI) counts are calculated, and primary miRNA mapping was performed using a humanspecific miRBase mature database. In the primary QIAseq quantification step, adapter sequences from the library preparation process and any low-quality bases are removed. UMI counts for each miRNA were used for differential expression analysis.

\section{MicroRNA Expression Analysis and Gene Ontology}

A total of 2,559 miRNA expression profiles at the baseline and 12 months were quantified in UMI counts. Cross-sectional upper normalized expression analysis for a single dose of placebo, $150 \mathrm{mg}, 200 \mathrm{mg}, 300 \mathrm{mg}$, or $400 \mathrm{mg}$ nilotinib ( $\mathrm{n}=$ 15) at baseline was conducted using the Kruskal-Wallis test. In the longitudinal phase, 2 analyses were performed in parallel. First, longitudinal differentially expressed miRNAs (DEMs) were determined via DESeq2 with the ratio of means normalization and followed by the Wald test ${ }^{22}$; second, relative and absolute changes in upper normalized expressions ${ }^{23}$ in the 3 treatment groups were compared using the KruskalWallis test. Mixed-effects analysis of variance (ME-ANOVA) was fitted to see whether there are associations between treatment and miRNA association cross-sectionally and longitudinally. Only DEMs that met both the Wald test and MEANOVA $p<0.05$ were used for further analysis. Gene targets were predicted in silico using the microRNA Target Filter tool in Ingenuity Pathway Analysis (Qiagen). Gene targets were filtered to include only highly predicted and experimentally supported mRNA target predictions. Gene ontology (GO) pathway and functional enrichment analyses were performed on the gene targets of the significant DEMs using the PANTHER Classification System. ${ }^{24}$ Only enriched pathways and GO terms that met Benjamini-Hochberg $p<0.05$ were reported.

\section{MicroRNA Correlations With Clinical Outcomes}

Correlation between 12-month longitudinal changes in UPDRS II-III and PDQ39 and absolute and relative longitudinal changes in miRNAs was computed using Spearman rank correlations, respectively.

\section{Post Hoc Power Analysis}

When $5 \%$ of miRNAs (125 among approximately 2,500 miRNA) are informative with $\log 2$ fold-change (FC) values indicating greater than 2 -fold expression changes, a mean count of 500, and a common dispersion of 0.1, 25 samples in each of 2 treatment arms would provide approximately $95 \%$ power at the false discovery rate of $0.05{ }^{25}$

\section{Data Availability}

The data supporting the current study are available from the corresponding author on request.

\section{Results}

\section{CSF miRNAs Are Not Altered After a Single Dose of Nilotinib}

miRNAs are dynamic regulators of gene expression and may play short- and long-term roles in gene expression. To assess whether miRNAs can be rapidly altered after a single dose of nilotinib, we performed whole-genome miRNA sequencing in the CSF of 75 patients with PD $(n=75)$ collected after a single oral dose of 150,200, 300, and $400 \mathrm{mg}$ nilotinib or placebo $(\mathrm{n}=15)($ Figure 1A). Expression profile analysis across all treatment groups shows no short-term effect of nilotinib on miRNA compared with placebo (eTable 1, links.lww.com/ NXG/A489). Only hsa-miR-451a was significantly altered (Kruskal-Wallis $p<0.05$ and FC $>2.0$ ) but appears to have no known associated gene targets according to miRTarBase (Version 8.0). ${ }^{26}$ miRNAs may not require large changes in expression to regulate miRNA targets; therefore, we evaluated all miRNAs that met $p<0.05$ (instead of FC) for treated vs untreated and dose-dependent effects. Even with these relaxed statistical thresholds, there were no miRNAs that show treatment effects compared with placebo after a single dose of nilotinib. In addition, principal component analysis was unable to distinguish treated samples from placebo (Figure 1B) or any distinct nilotinib treatment groups (Figure 1C).

\section{CSF miRNA Expression Is Differentially Altered in Treated vs Untreated Patients With PD}

Because we observed no differences in miRNA expression cross-sectionally between treatment groups and placebo after a single dose of nilotinib, we evaluated whether there were longitudinal changes in miRNA expression after 12 months of daily oral nilotinib, 150 or $300 \mathrm{mg}$, vs placebo. ${ }^{14,16,21}$ UMI counts for 2,559 miRNAs were mapped (Figure 1A) to the human genome in all samples, indicating detection and sequencing of the entire miRNA genome. The difference in absolute UMI counts between 12 months and baseline was plotted, and hierarchical clustering was performed using Morpheus (Broad Institute, Boston, MA) online tools (eFigure 1A, links.lww. com/NXG/A489). Longitudinal expression analysis to determine significantly DEM was performed in parallel using DESeq2 (Bioconductor) for ratio of means normalization followed by Wald testing and NOIseq (Bioconductor) for upper quantile normalization followed by ME-ANOVA with Kruskal-Wallis testing (eFigure 1B, links.lww.com/NXG/A489). Only 125 DEMs $(p<0.05)$ were found in common between both analyses (Table 1), in placebo (59 miRNAs) vs nilotinib, $150 \mathrm{mg}$ (27 miRNAs), and $300 \mathrm{mg}$ (43 miRNAs). Only 4 miRNAs were in significantly differentially expressed in 1 or more treatment groups (Figure 2A). DEM expression FC ( 12 months- 0 months) was transformed into fold-regulation $(\mathrm{FR})$ values $\left(\mathrm{FR}=\mathrm{FC}^{-1}\right)$, a 
Figure 1 Cross-sectional Analysis of miRNA After a Single Dose of Nilotinib

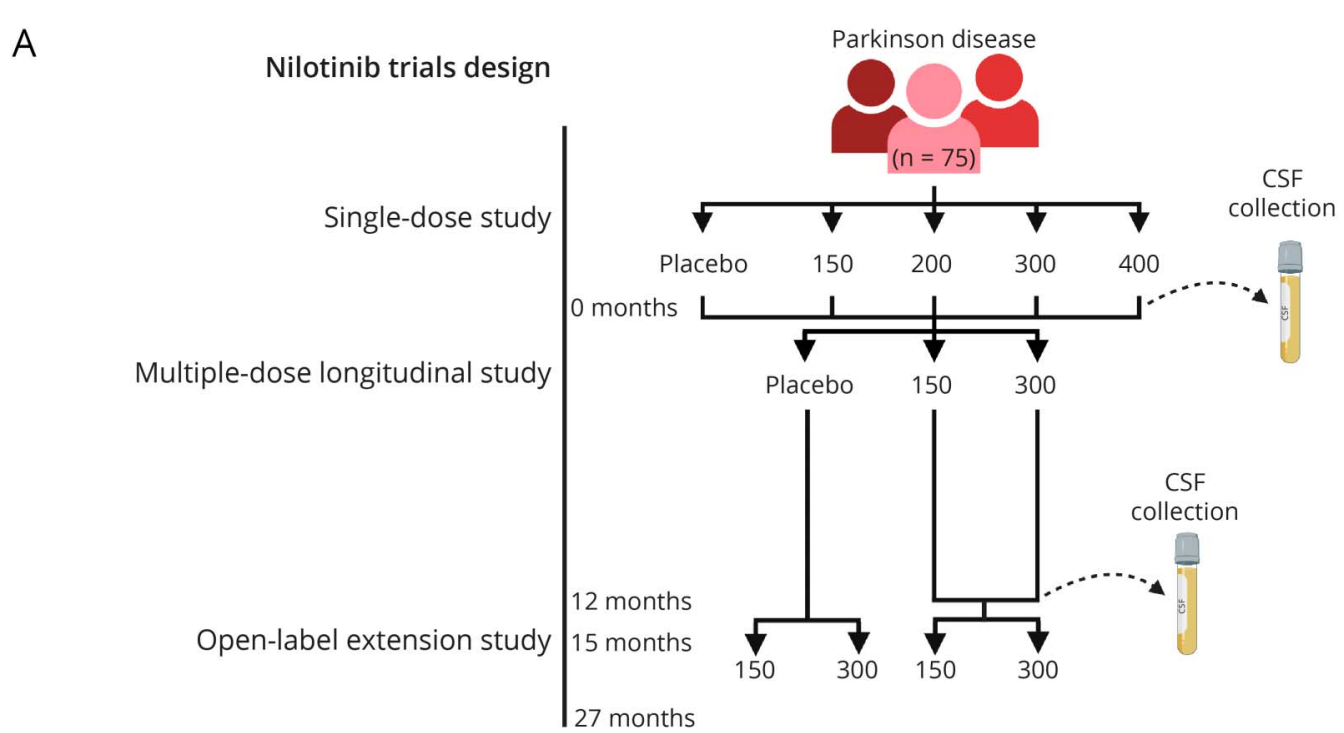

B

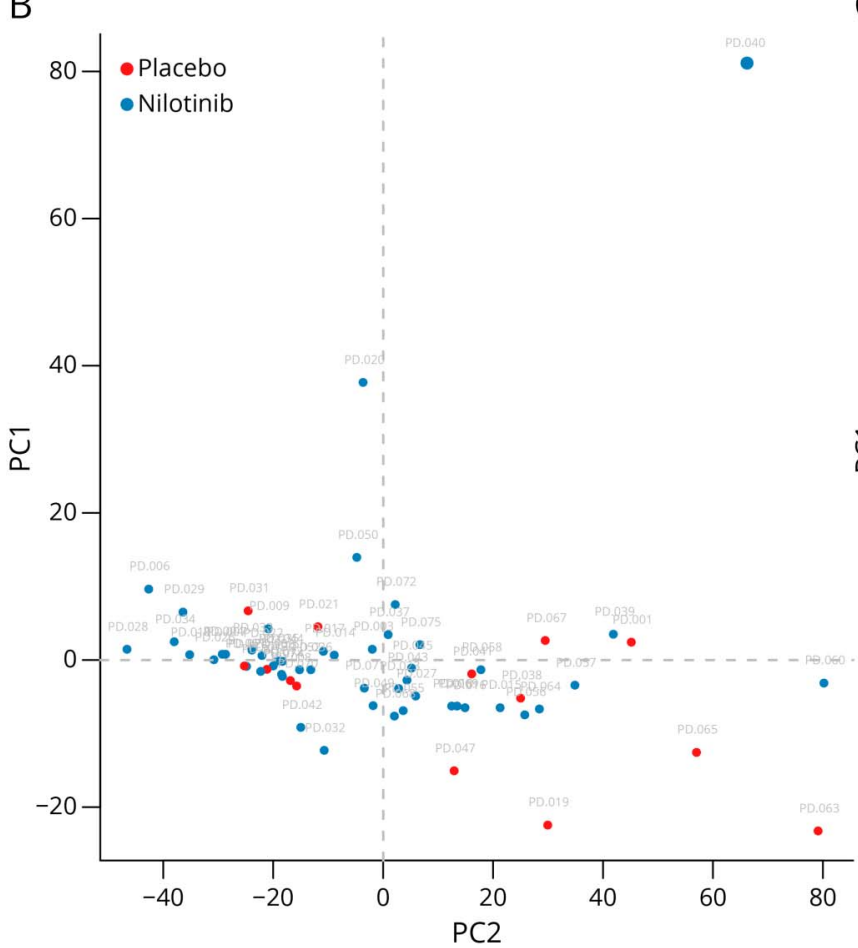

C

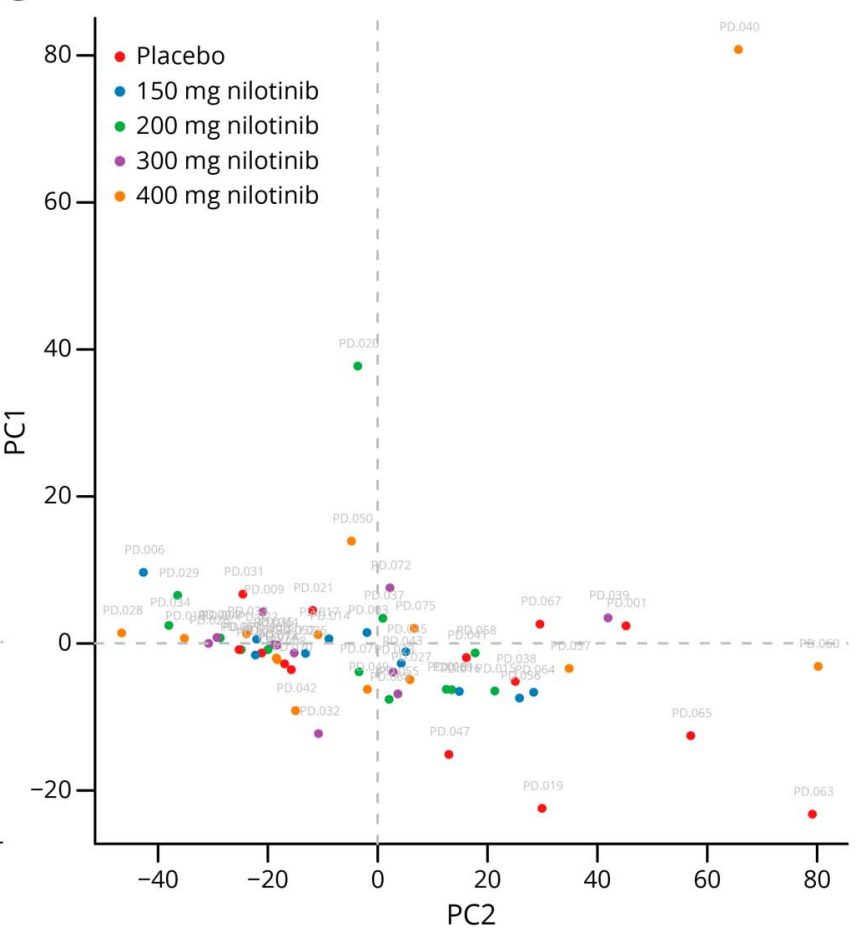

(A) Schematic of the clinical trial design. This schematic was created using BioRender.com. (B) Principal component analysis of miRNA expression in the singledose study for treatment vs untreated effects with placebo-(red) and nilotinib-(blue) treated populations and (C) placebo (red), $150 \mathrm{mg}$ (blue), $200 \mathrm{mg}$ (green), $300 \mathrm{mg}$ (purple), and $400 \mathrm{mg}$ (orange) nilotinib-treated populations. Principal component analysis classified 63 participants into groups using 2,559 miRNAs. PC1 and PC2 are depicted.

metric that better represents the directionality of longitudinal expression changes. The specific direction of longitudinal expression was the same between nilotinib, $150 \mathrm{mg}$ and $300 \mathrm{mg}$, groups and opposite to the placebo group, indicating treatment effects, as outlined in dashed lines and to the right of the heatmap (Figure 2B).

We searched known gene targets of the DEMs in each treatment group via Ingenuity Pathway Analysis (Qiagen). Of all possible TKs, we found SRC (hsa-miR-3918), which is not a known pharmacologic target of nilotinib, to be a predicted target of nilotinib, $150 \mathrm{mg}$, and DDR1 (hsa-miR-5195-3p), which is the preferred target of nilotinib, as a predicted target of $300 \mathrm{mg}$. Both of these TKs were the targets of miRNAs with decreased expression, suggesting disinhibition and potential subsequent expression of SRC and DDR1 with nilotinib, $150 \mathrm{mg}$ and $300 \mathrm{mg}$, respectively. Although nilotinib acts on the protein to dephosphorylate and inhibit DDR1, it 
Table 1 Longitudinal Differential Expression of 125 miRNAs

\begin{tabular}{|c|c|c|c|c|c|c|c|c|c|}
\hline & Placebo & & & $\begin{array}{l}150 \mathrm{mg} \\
\text { nilotinib }\end{array}$ & & & $\begin{array}{l}300 \mathrm{mg} \\
\text { nilotinib }\end{array}$ & & \\
\hline miRNA & $\mathrm{FR}$ & Wald test $p$ & ME-ANOVA $p$ & $\mathrm{FR}$ & Wald test $p$ & ME-ANOVA $p$ & $\mathrm{FR}$ & Wald test $p$ & ME-ANOVA $p$ \\
\hline hsa-let-7a-3p & -1.08 & n.s. & n.s. & -1.31 & 7.91E-03 & $3.61 \mathrm{E}-02$ & 1.02 & n.s. & n.s. \\
\hline hsa-let-7i-3p & -1.24 & $1.36 \mathrm{E}-02$ & 4.75E-02 & -1.22 & $2.44 \mathrm{E}-02$ & n.s. & -1.09 & n.s. & n.s. \\
\hline hsa-miR-105-3p & 1.16 & n.s. & n.s. & 1.26 & $1.26 \mathrm{E}-03$ & $8.81 \mathrm{E}-03$ & 1.02 & n.s. & n.s. \\
\hline hsa-miR-1178-3p & -1.14 & n.s. & n.s. & 1.07 & n.s. & n.s. & 1.13 & $3.90 \mathrm{E}-02$ & $2.24 \mathrm{E}-02$ \\
\hline hsa-miR-1245b-5p & 1.33 & 8.93E-03 & $3.50 \mathrm{E}-02$ & -1.05 & n.s. & $2.99 \mathrm{E}-02$ & 1.02 & n.s. & n.s. \\
\hline hsa-miR-1251-5p & -1.13 & $1.71 \mathrm{E}-02$ & $3.70 \mathrm{E}-03$ & -1.14 & $1.93 \mathrm{E}-02$ & n.s. & -1.07 & n.s. & n.s. \\
\hline hsa-miR-1253 & -1.03 & n.s. & $3.66 \mathrm{E}-02$ & 1.17 & 4.87E-02 & $2.11 \mathrm{E}-02$ & -1.03 & n.s. & n.s. \\
\hline hsa-miR-1268a & 1.17 & $2.46 \mathrm{E}-02$ & $8.38 \mathrm{E}-03$ & 1.14 & n.s. & n.s. & 1.11 & n.s. & n.s. \\
\hline hsa-miR-128-1-5p & 1.28 & $8.66 \mathrm{E}-04$ & $1.57 \mathrm{E}-02$ & 1.11 & n.s. & n.s. & 1.05 & n.s. & n.s. \\
\hline hsa-miR-1306-5p & -1.24 & n.s. & n.s. & -1.07 & n.s. & n.s. & 1.34 & $4.48 \mathrm{E}-03$ & $1.32 \mathrm{E}-02$ \\
\hline hsa-miR-145-3p & 1.08 & n.s. & $3.73 \mathrm{E}-02$ & -1.28 & 7.94E-03 & $1.71 \mathrm{E}-03$ & 1.17 & n.s. & n.s. \\
\hline hsa-miR-1587 & -1.09 & n.s. & n.s. & 1.12 & $3.81 \mathrm{E}-02$ & $2.86 \mathrm{E}-02$ & -1.05 & n.s. & n.s. \\
\hline hsa-miR-15a-5p & 1.05 & n.s. & n.s. & -1.01 & n.s. & n.s. & -1.19 & $1.67 \mathrm{E}-03$ & $1.54 \mathrm{E}-02$ \\
\hline hsa-miR-182-5p & -1.05 & n.s. & n.s. & 1.14 & n.s. & n.s. & 1.25 & $1.67 \mathrm{E}-02$ & $9.96 \mathrm{E}-03$ \\
\hline hsa-miR-18b-3p & -1.24 & $2.68 \mathrm{E}-02$ & $3.69 \mathrm{E}-02$ & 1.06 & n.s. & n.s. & -1.04 & n.s. & n.s. \\
\hline hsa-miR-1910-3p & 1.01 & n.s. & n.s. & -1.22 & $2.88 \mathrm{E}-02$ & $4.06 \mathrm{E}-02$ & -1.08 & n.s. & n.s. \\
\hline hsa-miR-193a-5p & -1.07 & n.s. & $3.79 \mathrm{E}-02$ & 1.26 & $3.14 \mathrm{E}-02$ & $6.03 E-03$ & -1.07 & n.s. & n.s. \\
\hline hsa-miR-212-5p & -1.11 & $3.46 \mathrm{E}-02$ & $3.30 \mathrm{E}-02$ & -1.02 & n.s. & n.s. & -1.21 & $2.87 \mathrm{E}-05$ & n.s. \\
\hline hsa-miR-217 & -1.07 & n.s. & n.s. & -1.03 & n.s. & n.s. & -1.2 & $8.34 \mathrm{E}-03$ & $4.43 \mathrm{E}-02$ \\
\hline hsa-miR-26a-1-3p & -1.22 & $3.16 \mathrm{E}-02$ & 4.46E-02 & -1.02 & n.s. & n.s. & 1.36 & $1.53 \mathrm{E}-03$ & $1.90 \mathrm{E}-03$ \\
\hline hsa-miR-28-5p & -1.27 & 4.62E-02 & $1.82 \mathrm{E}-03$ & 1.04 & n.s. & $1.74 \mathrm{E}-03$ & -1.19 & n.s. & n.s. \\
\hline hsa-miR-297 & 1.05 & n.s. & 4.09E-02 & -1.22 & $6.61 \mathrm{E}-03$ & $1.91 \mathrm{E}-02$ & 1 & n.s. & $4.12 \mathrm{E}-02$ \\
\hline hsa-miR-30b-5p & -1.13 & $2.90 \mathrm{E}-02$ & $3.79 \mathrm{E}-02$ & 1.05 & n.s. & n.s. & 1.02 & n.s. & n.s. \\
\hline hsa-miR-3133 & -1.07 & n.s. & n.s. & -1.26 & $6.25 \mathrm{E}-03$ & n.s. & 1.21 & $1.88 \mathrm{E}-02$ & $2.12 \mathrm{E}-02$ \\
\hline hsa-miR-3153 & 1.17 & $2.39 \mathrm{E}-02$ & 4.97E-02 & -1.01 & n.s. & n.s. & 1.06 & n.s. & n.s. \\
\hline hsa-miR-3155a & -1.12 & $3.27 \mathrm{E}-02$ & $2.49 \mathrm{E}-02$ & -1.03 & n.s. & n.s. & -1.19 & 7.34E-03 & n.s. \\
\hline hsa-miR-3166 & 1.15 & $2.69 \mathrm{E}-02$ & $2.45 \mathrm{E}-02$ & -1.04 & n.s. & $1.40 \mathrm{E}-02$ & -1.04 & n.s. & n.s. \\
\hline hsa-miR-3169 & -1.02 & n.s. & n.s. & -1.05 & n.s. & n.s. & -1.18 & $9.38 \mathrm{E}-04$ & $2.50 \mathrm{E}-02$ \\
\hline hsa-miR-328-3p & -1.21 & n.s. & n.s. & 1.13 & n.s. & n.s. & 1.37 & $1.87 \mathrm{E}-04$ & $4.91 \mathrm{E}-03$ \\
\hline hsa-miR-346 & 1.12 & n.s. & $6.52 \mathrm{E}-03$ & 1.22 & n.s. & $1.52 \mathrm{E}-02$ & 1.29 & $4.31 \mathrm{E}-02$ & $1.04 \mathrm{E}-02$ \\
\hline hsa-miR-3618 & 1.07 & n.s. & n.s. & -1.2 & $6.78 \mathrm{E}-03$ & $1.55 \mathrm{E}-02$ & 1.05 & n.s. & n.s. \\
\hline hsa-miR-3661 & 1.03 & n.s. & n.s. & 1.06 & n.s. & n.s. & -1.15 & $2.05 \mathrm{E}-02$ & $3.87 \mathrm{E}-02$ \\
\hline hsa-miR-3668 & -1 & n.s. & n.s. & 1.01 & n.s. & n.s. & -1.16 & $1.99 \mathrm{E}-03$ & $3.19 \mathrm{E}-02$ \\
\hline hsa-miR-3684 & -1.06 & n.s. & n.s. & -1.07 & n.s. & n.s. & -1.2 & 3.49E-04 & $4.31 \mathrm{E}-02$ \\
\hline hsa-miR-378c & -1.17 & n.s. & n.s. & 1.03 & n.s. & n.s. & -1.23 & $3.71 \mathrm{E}-02$ & $1.96 \mathrm{E}-02$ \\
\hline hsa-miR-378e & -1.16 & n.s. & n.s. & 1.33 & $2.78 \mathrm{E}-03$ & $1.61 \mathrm{E}-02$ & -1.01 & n.s. & n.s. \\
\hline hsa-miR-382-5p & -1.18 & $1.74 \mathrm{E}-02$ & $1.80 \mathrm{E}-02$ & 1.01 & n.s. & n.s. & 1.05 & n.s. & 4.99E-02 \\
\hline
\end{tabular}


Table 1 Longitudinal Differential Expression of 125 miRNAs (continued)

\begin{tabular}{|c|c|c|c|c|c|c|c|c|c|}
\hline \multirow[b]{2}{*}{ hsa-miR-3918 } & \multicolumn{3}{|c|}{ Placebo } & \multicolumn{3}{|c|}{$\begin{array}{l}150 \mathrm{mg} \\
\text { nilotinib }\end{array}$} & \multicolumn{3}{|c|}{$\begin{array}{l}300 \mathrm{mg} \\
\text { nilotinib }\end{array}$} \\
\hline & 1.1 & n.s. & n.s. & -1.19 & $2.86 \mathrm{E}-02$ & $3.65 \mathrm{E}-02$ & -1.14 & n.s. & n.s. \\
\hline hsa-miR-4252 & -1.24 & $1.72 \mathrm{E}-02$ & $1.69 \mathrm{E}-02$ & 1.09 & n.s. & n.s. & 1.06 & n.s. & $1.06 \mathrm{E}-02$ \\
\hline hsa-miR-4312 & 1.05 & n.s. & n.s. & -1.26 & $8.20 \mathrm{E}-03$ & $4.27 \mathrm{E}-02$ & 1.34 & $6.99 \mathrm{E}-03$ & n.s. \\
\hline hsa-miR-4313 & -1.19 & n.s. & n.s. & 1.05 & n.s. & n.s. & 1.33 & $1.60 \mathrm{E}-02$ & $3.78 \mathrm{E}-02$ \\
\hline hsa-miR-4328 & 1.21 & $4.82 \mathrm{E}-02$ & $4.56 \mathrm{E}-02$ & 1.06 & n.s. & n.s. & -1.16 & $4.21 \mathrm{E}-02$ & $3.70 \mathrm{E}-03$ \\
\hline hsa-miR-4435 & -1.24 & $1.12 \mathrm{E}-02$ & $1.88 \mathrm{E}-03$ & -1.14 & n.s. & n.s. & 1.16 & n.s. & $1.20 \mathrm{E}-03$ \\
\hline hsa-miR-4436b-3p & -1.14 & n.s. & n.s. & 1.25 & $7.08 \mathrm{E}-03$ & $3.50 \mathrm{E}-03$ & 1.18 & n.s. & n.s. \\
\hline hsa-miR-4441 & 1.24 & $3.25 \mathrm{E}-02$ & $3.62 \mathrm{E}-02$ & -1.01 & n.s. & n.s. & 1.06 & n.s. & n.s. \\
\hline hsa-miR-4455 & 1.46 & $1.75 \mathrm{E}-03$ & $2.57 \mathrm{E}-02$ & 1.04 & n.s. & $3.46 \mathrm{E}-03$ & -1.07 & n.s. & $6.85 E-03$ \\
\hline hsa-miR-4464 & -1.04 & n.s. & n.s. & 1.15 & $4.73 E-02$ & $4.42 \mathrm{E}-02$ & 1.14 & n.s. & n.s. \\
\hline hsa-miR-4469 & -1.13 & $1.89 \mathrm{E}-02$ & $2.91 \mathrm{E}-02$ & -1.06 & n.s. & n.s. & 1.15 & n.s. & n.s. \\
\hline hsa-miR-4490 & 1.2 & $2.61 \mathrm{E}-02$ & 4.13E-02 & -1.02 & n.s. & $2.20 \mathrm{E}-02$ & -1.02 & n.s. & $2.27 \mathrm{E}-02$ \\
\hline hsa-miR-449c-3p & 1.17 & n.s. & n.s. & 1.16 & n.s. & n.s. & -1.27 & $1.67 \mathrm{E}-02$ & $2.27 \mathrm{E}-02$ \\
\hline hsa-miR-4508 & -1.09 & n.s. & $2.51 \mathrm{E}-02$ & 1.27 & $1.33 E-02$ & $2.80 \mathrm{E}-03$ & 1.2 & n.s. & n.s. \\
\hline hsa-miR-4527 & -1.18 & $5.00 \mathrm{E}-03$ & $3.50 \mathrm{E}-03$ & -1.09 & n.s. & n.s. & -1.22 & $1.76 \mathrm{E}-05$ & n.s. \\
\hline hsa-miR-4632-3p & 1.28 & $1.27 \mathrm{E}-02$ & $1.42 \mathrm{E}-02$ & -1.08 & n.s. & n.s. & 1.1 & n.s. & n.s. \\
\hline hsa-miR-4636 & 1.32 & $1.11 \mathrm{E}-02$ & $3.22 \mathrm{E}-02$ & -1.05 & n.s. & n.s. & -1.2 & n.s. & n.s. \\
\hline hsa-miR-4666a-5p & -1.33 & $3.31 \mathrm{E}-03$ & n.s. & -1.1 & n.s. & n.s. & -1.28 & $5.23 E-03$ & $4.46 \mathrm{E}-02$ \\
\hline hsa-miR-4667-3p & -1.21 & $4.30 \mathrm{E}-02$ & $2.46 \mathrm{E}-02$ & 1.03 & n.s. & $8.08 \mathrm{E}-03$ & 1.4 & $4.13 E-03$ & 4.87E-02 \\
\hline hsa-miR-4683 & -1.04 & n.s. & n.s. & -1.13 & n.s. & n.s. & 1.32 & $8.65 \mathrm{E}-04$ & $3.72 \mathrm{E}-02$ \\
\hline hsa-miR-4685-5p & 1.26 & $8.31 \mathrm{E}-04$ & $5.82 \mathrm{E}-03$ & 1.06 & n.s. & n.s. & -1.01 & n.s. & $3.33 \mathrm{E}-02$ \\
\hline hsa-miR-4686 & -1.17 & $7.02 \mathrm{E}-04$ & $1.52 \mathrm{E}-03$ & -1.03 & n.s. & $4.61 \mathrm{E}-02$ & -1.19 & $1.90 \mathrm{E}-04$ & n.s. \\
\hline hsa-miR-4704-5p & 1.27 & $1.25 \mathrm{E}-02$ & $2.24 \mathrm{E}-02$ & -1.13 & n.s. & $3.85 \mathrm{E}-02$ & 1.18 & $1.54 \mathrm{E}-02$ & n.s. \\
\hline hsa-miR-4707-3p & -1.03 & n.s. & n.s. & 1.22 & $3.07 E-02$ & $1.61 \mathrm{E}-02$ & -1.03 & n.s. & n.s. \\
\hline hsa-miR-4712-5p & 1.22 & $1.92 \mathrm{E}-02$ & $3.52 \mathrm{E}-02$ & -1.22 & $6.37 \mathrm{E}-03$ & $2.54 \mathrm{E}-04$ & -1.05 & n.s. & $4.83 \mathrm{E}-02$ \\
\hline hsa-miR-4755-5p & -1.16 & $3.99 \mathrm{E}-02$ & $1.08 \mathrm{E}-02$ & -1.11 & n.s. & n.s. & -1.09 & n.s. & n.s. \\
\hline hsa-miR-4760-3p & -1.22 & $8.61 \mathrm{E}-03$ & $2.45 \mathrm{E}-02$ & 1.09 & n.s. & $2.42 \mathrm{E}-02$ & 1.03 & n.s. & n.s. \\
\hline hsa-miR-4793-3p & 1.17 & $2.40 \mathrm{E}-02$ & $1.77 \mathrm{E}-02$ & 1.08 & n.s. & $3.43 \mathrm{E}-02$ & 1.17 & n.s. & n.s. \\
\hline hsa-miR-4795-5p & -1.05 & n.s. & n.s. & -1.19 & $3.34 \mathrm{E}-02$ & $1.44 \mathrm{E}-02$ & -1.04 & n.s. & n.s. \\
\hline hsa-miR-4797-3p & -1.03 & n.s. & $4.41 \mathrm{E}-02$ & 1.32 & $3.77 \mathrm{E}-03$ & $4.65 \mathrm{E}-02$ & -1.11 & n.s. & n.s. \\
\hline hsa-miR-494-5p & 1.02 & n.s. & n.s. & -1.01 & n.s. & n.s. & -1.21 & $1.10 \mathrm{E}-03$ & $3.40 \mathrm{E}-02$ \\
\hline hsa-miR-5088-5p & 1.18 & $1.96 \mathrm{E}-02$ & 4.97E-02 & -1.01 & n.s. & n.s. & -1.09 & n.s. & 4.67E-02 \\
\hline hsa-miR-5094 & -1.02 & n.s. & n.s. & 1.03 & n.s. & n.s. & 1.16 & $2.66 \mathrm{E}-02$ & $4.20 \mathrm{E}-02$ \\
\hline hsa-miR-510-3p & -1.58 & $2.65 \mathrm{E}-05$ & $2.83 \mathrm{E}-02$ & -1.24 & $1.72 \mathrm{E}-02$ & n.s. & -1.3 & $1.52 \mathrm{E}-02$ & n.s. \\
\hline hsa-miR-5191 & 1.13 & $2.91 \mathrm{E}-02$ & $1.28 \mathrm{E}-02$ & 1.03 & n.s. & n.s. & -1.03 & n.s. & n.s. \\
\hline hsa-miR-5195-3p & 1.04 & n.s. & n.s. & -1.02 & n.s. & n.s. & -1.2 & $6.80 \mathrm{E}-03$ & $4.02 \mathrm{E}-02$ \\
\hline hsa-miR-548ad-5p & -1.33 & $4.86 \mathrm{E}-02$ & $1.15 \mathrm{E}-02$ & -1.09 & n.s. & n.s. & -1.66 & $1.78 \mathrm{E}-04$ & n.s. \\
\hline
\end{tabular}


Table 1 Longitudinal Differential Expression of 125 miRNAs (continued)

\begin{tabular}{|c|c|c|c|c|c|c|c|c|c|}
\hline \multirow[b]{2}{*}{ hsa-miR-548ar-5p } & \multicolumn{3}{|c|}{ Placebo } & \multicolumn{3}{|c|}{$\begin{array}{l}150 \mathrm{mg} \\
\text { nilotinib }\end{array}$} & \multicolumn{3}{|c|}{$\begin{array}{l}300 \mathrm{mg} \\
\text { nilotinib }\end{array}$} \\
\hline & 1.01 & n.s. & n.s. & -1.14 & n.s. & n.s. & -1.42 & $3.18 \mathrm{E}-02$ & $1.48 \mathrm{E}-02$ \\
\hline hsa-miR-548as-5p & -1.21 & $9.31 \mathrm{E}-03$ & $4.90 \mathrm{E}-02$ & 1.09 & n.s. & n.s. & 1 & n.s. & 3.77E-02 \\
\hline hsa-miR-548az-5p & 1.11 & n.s. & n.s. & 1.12 & n.s. & n.s. & -1.14 & $1.50 \mathrm{E}-02$ & $2.16 \mathrm{E}-03$ \\
\hline hsa-miR-5571-5p & 1.11 & n.s. & n.s. & -1.13 & $2.85 \mathrm{E}-02$ & $2.36 \mathrm{E}-02$ & -1.02 & n.s. & n.s. \\
\hline hsa-miR-5579-5p & 1.02 & n.s. & n.s. & -1.16 & n.s. & n.s. & -1.2 & $2.59 \mathrm{E}-02$ & $3.59 \mathrm{E}-02$ \\
\hline hsa-miR-5586-3p & 1.19 & $2.93 \mathrm{E}-02$ & n.s. & 1.03 & n.s. & n.s. & -1.25 & $1.46 \mathrm{E}-02$ & $3.26 \mathrm{E}-02$ \\
\hline hsa-miR-5588-5p & 1.18 & $3.05 E-02$ & $3.05 E-02$ & -1.06 & n.s. & n.s. & 1.15 & n.s. & n.s. \\
\hline hsa-miR-5590-5p & -1.06 & n.s. & n.s. & -1.05 & n.s. & n.s. & -1.28 & $1.80 \mathrm{E}-03$ & $1.45 \mathrm{E}-02$ \\
\hline hsa-miR-5591-3p & 1.16 & $3.20 \mathrm{E}-02$ & $3.85 \mathrm{E}-02$ & 1.04 & n.s. & n.s. & -1 & n.s. & n.s. \\
\hline hsa-miR-5682 & 1.03 & n.s. & n.s. & 1.06 & n.s. & n.s. & -1.23 & $6.82 \mathrm{E}-03$ & 4.97E-02 \\
\hline hsa-miR-591 & 1.21 & n.s. & n.s. & -1.16 & n.s. & n.s. & -1.32 & $1.20 \mathrm{E}-02$ & $2.97 \mathrm{E}-02$ \\
\hline hsa-miR-6076 & -1.14 & $3.77 \mathrm{E}-02$ & $2.24 \mathrm{E}-02$ & -1.09 & n.s. & n.s. & -1.2 & $2.27 \mathrm{E}-03$ & n.s. \\
\hline hsa-miR-6080 & 1 & n.s. & n.s. & 1.34 & $9.45 \mathrm{E}-04$ & $3.58 \mathrm{E}-02$ & -1.04 & n.s. & n.s. \\
\hline hsa-miR-6084 & 1.1 & n.s. & n.s. & 1.08 & n.s. & n.s. & -1.25 & $4.25 E-02$ & $3.50 \mathrm{E}-03$ \\
\hline hsa-miR-6090 & -1.09 & n.s. & n.s. & -1.01 & n.s. & n.s. & 1.31 & $4.50 \mathrm{E}-03$ & $2.61 \mathrm{E}-02$ \\
\hline hsa-miR-615-5p & -1.2 & $1.82 \mathrm{E}-02$ & $3.38 \mathrm{E}-03$ & -1.01 & n.s. & n.s. & -1.02 & n.s. & n.s. \\
\hline hsa-miR-625-3p & -1.44 & $1.00 \mathrm{E}-03$ & $3.08 \mathrm{E}-03$ & -1.09 & n.s. & n.s. & 1.02 & n.s. & n.s. \\
\hline hsa-miR-6508-5p & 1.23 & $1.67 \mathrm{E}-02$ & n.s. & 1.1 & n.s. & n.s. & 1.24 & $3.68 \mathrm{E}-02$ & 3.37E-02 \\
\hline hsa-miR-6513-3p & 1.17 & $2.25 \mathrm{E}-02$ & $3.47 \mathrm{E}-02$ & -1.07 & n.s. & 1.97E-02 & 1.13 & n.s. & n.s. \\
\hline hsa-miR-6716-3p & -1.06 & n.s. & n.s. & -1.01 & n.s. & n.s. & -1.13 & $7.93 \mathrm{E}-03$ & $2.52 \mathrm{E}-02$ \\
\hline hsa-miR-6724-5p & -1.17 & $2.57 \mathrm{E}-02$ & $1.13 E-02$ & -1.02 & n.s. & n.s. & -1.03 & n.s. & n.s. \\
\hline hsa-miR-6733-5p & 1.12 & n.s. & n.s. & -1.19 & $1.86 \mathrm{E}-02$ & $9.46 \mathrm{E}-03$ & 1.1 & n.s. & n.s. \\
\hline hsa-miR-6735-5p & 1.09 & n.s. & n.s. & -1.17 & 4.67E-02 & $9.27 \mathrm{E}-03$ & 1.09 & n.s. & n.s. \\
\hline hsa-miR-6736-3p & -1.03 & n.s. & n.s. & 1.62 & 5.67E-05 & $1.78 \mathrm{E}-02$ & -1.31 & $3.33 E-02$ & n.s. \\
\hline hsa-miR-6748-3p & -1.26 & $7.12 \mathrm{E}-03$ & $3.63 \mathrm{E}-02$ & 1.18 & n.s. & n.s. & 1.12 & n.s. & n.s. \\
\hline hsa-miR-6757-5p & -1.03 & n.s. & n.s. & 1.11 & $4.53 \mathrm{E}-02$ & $4.78 \mathrm{E}-02$ & -1.04 & n.s. & n.s. \\
\hline hsa-miR-6758-3p & 1.16 & $3.03 E-02$ & $7.43 \mathrm{E}-03$ & 1.19 & $1.16 \mathrm{E}-02$ & n.s. & 1.22 & $2.74 \mathrm{E}-03$ & n.s. \\
\hline hsa-miR-6766-5p & -1.14 & $1.17 \mathrm{E}-02$ & $3.02 \mathrm{E}-02$ & -1.05 & n.s. & n.s. & -1.02 & n.s. & $2.49 \mathrm{E}-02$ \\
\hline hsa-miR-6788-3p & 1.12 & $4.19 E-02$ & $4.14 \mathrm{E}-02$ & 1.01 & n.s. & n.s. & 1.02 & n.s. & n.s. \\
\hline hsa-miR-6808-3p & -1.24 & $2.31 \mathrm{E}-02$ & 4.37E-03 & 1.01 & n.s. & $2.58 \mathrm{E}-02$ & 1.02 & n.s. & n.s. \\
\hline hsa-miR-6813-5p & 1.08 & n.s. & n.s. & 1.04 & n.s. & n.s. & -1.17 & $2.29 \mathrm{E}-02$ & $3.42 \mathrm{E}-03$ \\
\hline hsa-miR-6821-3p & 1.15 & $1.97 \mathrm{E}-02$ & $3.06 \mathrm{E}-02$ & -1.02 & n.s. & n.s. & 1.03 & n.s. & n.s. \\
\hline hsa-miR-6832-5p & 1.02 & n.s. & n.s. & -1.15 & n.s. & n.s. & 1.31 & $1.74 \mathrm{E}-04$ & $3.66 \mathrm{E}-02$ \\
\hline hsa-miR-6861-5p & 1.09 & n.s. & n.s. & 1.06 & n.s. & n.s. & -1.31 & $4.40 \mathrm{E}-03$ & $1.44 \mathrm{E}-02$ \\
\hline hsa-miR-6875-5p & 1.17 & n.s. & n.s. & 1.13 & n.s. & n.s. & -1.19 & $1.78 \mathrm{E}-03$ & $3.86 \mathrm{E}-02$ \\
\hline hsa-miR-6876-5p & -1.15 & $4.08 \mathrm{E}-02$ & $1.68 \mathrm{E}-02$ & 1.03 & n.s. & n.s. & -1.03 & n.s. & n.s. \\
\hline hsa-miR-6890-3p & -1.06 & n.s. & n.s. & -1.09 & n.s. & n.s. & 1.29 & $3.79 E-02$ & $2.64 \mathrm{E}-02$ \\
\hline
\end{tabular}


Table 1 Longitudinal Differential Expression of 125 miRNAs (continued)

\begin{tabular}{|c|c|c|c|c|c|c|c|c|c|}
\hline & Placebo & & & $\begin{array}{l}150 \mathrm{mg} \\
\text { nilotini }\end{array}$ & & & $\begin{array}{l}300 \mathrm{mg} \\
\text { nilotinib }\end{array}$ & & \\
\hline hsa-miR-7106-3p & -1.2 & $3.36 \mathrm{E}-02$ & $1.07 \mathrm{E}-02$ & -1.04 & n.s. & n.s. & 1.03 & n.s. & n.s. \\
\hline hsa-miR-7113-3p & -1.28 & $8.41 \mathrm{E}-04$ & $3.49 \mathrm{E}-03$ & -1.1 & n.s. & n.s. & -1.12 & n.s. & n.s. \\
\hline hsa-miR-7150 & -1.07 & n.s. & n.s. & -1.13 & n.s. & n.s. & -1.25 & $1.86 \mathrm{E}-03$ & $4.11 \mathrm{E}-02$ \\
\hline hsa-miR-7156-5p & -1.04 & n.s. & n.s. & 1.11 & n.s. & n.s. & 1.43 & 3.93E-04 & $1.56 \mathrm{E}-02$ \\
\hline hsa-miR-7162-3p & 1.15 & n.s. & n.s. & 1.11 & n.s. & n.s. & 1.39 & $3.66 \mathrm{E}-02$ & $1.85 \mathrm{E}-02$ \\
\hline hsa-miR-7162-5p & -1.11 & $3.92 \mathrm{E}-02$ & $4.82 \mathrm{E}-02$ & 1.04 & n.s. & n.s. & -1.07 & n.s. & n.s. \\
\hline hsa-miR-7706 & 1.2 & $1.59 \mathrm{E}-02$ & $4.15 \mathrm{E}-02$ & 1.03 & n.s. & n.s. & -1.01 & n.s. & n.s. \\
\hline hsa-miR-7853-5p & -1.38 & $2.66 \mathrm{E}-03$ & $4.79 E-02$ & -1.14 & n.s. & n.s. & -1.25 & 4.25E-02 & n.s. \\
\hline hsa-miR-7973 & 1.33 & $9.47 \mathrm{E}-03$ & $2.00 \mathrm{E}-02$ & 1.02 & n.s. & n.s. & 1.1 & n.s. & n.s. \\
\hline hsa-miR-8079 & -1.28 & $4.88 \mathrm{E}-03$ & $1.44 \mathrm{E}-02$ & -1 & n.s. & $1.90 \mathrm{E}-02$ & -1 & n.s. & n.s. \\
\hline hsa-miR-8083 & -1.01 & n.s. & n.s. & 1.14 & $2.47 \mathrm{E}-02$ & $4.00 \mathrm{E}-02$ & -1.01 & n.s. & n.s. \\
\hline hsa-miR-873-3p & 1.02 & n.s. & n.s. & -1.08 & n.s. & n.s. & -1.18 & $4.38 \mathrm{E}-03$ & $4.38 \mathrm{E}-02$ \\
\hline hsa-miR-873-5p & 1.06 & n.s. & n.s. & -1.33 & $7.20 \mathrm{E}-03$ & $3.39 \mathrm{E}-02$ & -1.06 & n.s. & n.s. \\
\hline hsa-miR-889-5p & 1.19 & 1.77E-03 & $1.30 \mathrm{E}-02$ & 1.08 & n.s. & n.s. & 1.22 & $4.60 \mathrm{E}-04$ & n.s. \\
\hline
\end{tabular}

Abbreviations: $\mathrm{FR}=$ fold regulation (fold-change ${ }^{-1}$ ); ME-ANOVA = mixed-effects analysis of variance; $\mathrm{n} . \mathrm{s}$. $=$ not significant $(p>0.05)$. $p$ Values calculated for within-group longitudinal expression changes using the Wald test and within- and between-group longitudinal expression changes using ME-ANOVA (not corrected for multiple testing).

may be that sustained inhibition of TKs at the level of the protein alters expression patterns of the genes.

\section{Longitudinal Alterations of miRNAs That Regulate Autophagy}

Key autophagy genes ATG12 (hsa-miR-30b-5p), ATG5 (hsamiR-30b-5p) BECN1 (hsa-miR-30b-5p), and MAP1LC3C (hsa-miR-4252) are gene targets associated with autophagy, as predicted using Ingenuity Pathway Analysis software (Figure 2D). These miRNAs are significantly decreased in patients with moderately severe PD in the placebo group between baseline and 12 months, indicating an increase in the expression of autophagy genes, suggesting possible changes of autophagy in the progression of PD. These genes were shown to change in animal models treated with nilotinib ${ }^{9-16}$; these genes are well characterized for their roles in the initiation and early stages of autophagosome construction for macroautophagy and chaperone-mediated autophagy. ${ }^{27}$ An increase in ATG5 and conjugated ATG12 suggests initiation of autophagy, but expression of MAPLC3C indicates accumulation of autophagosomes. ${ }^{8,9,13,27}$ Moreover, lysosomeassociated membrane protein (LAMP)-1, a component of the lysosome membrane, is the target of hsa-miR-3166, whose expression is significantly increased in placebo-treated patients, suggesting reduction of this lysosomal protein. Together, this pattern of disinhibited and suppressed genes suggests an attempt of neurons to execute autophagic flux and clearance $^{27}$ over 1 year, consistent with animal data ${ }^{8,9}$ and postmortem human brains ${ }^{2,28}$ showing accumulation of undigested autophagic vacuoles.

\section{Nilotinib Differentially Alters miRNAs That Regulate Autophagy}

Although there are some autophagy genes associated with the DEMs in the nilotinib, $150 \mathrm{mg}$ group, nilotinib, $300 \mathrm{mg}$ vs placebo presents a pattern of gene targets along the entire autophagy-lysosome pathway. Here, we observe inhibition of hsa-miR-15a-5p, which targets genes associated with regulation of BCL2 and ATG9A, suggesting autophagosome maturation (Figure 2D). Together, an increase in ATG9 expression and disappearance of MAP1LC3 indicate autophagosome clearance. ${ }^{8,9,13,27}$ The expression of hsa-miR5195, which targets LAMP2 expression, was also reduced. Although LAMP1 and LAMP2 proteins share many similarities and responsibilities, LAMP2 mediates maturation of the autophagosome, and its loss leads to increases in phagophore accumulation, whereas loss of LAMP1 is associated with inhibition of autophagy. ${ }^{29,30}$ Taken together, these data indicate that nilotinib, $300 \mathrm{mg}$, reverses the autophagic defects observed in placebo.

\section{Nilotinib Regulates miRNAs Associated With (De)ubiquitination}

Changes of miRNAs associated with the ubiquitination pathways were detected only in nilotinib, $300 \mathrm{vs} 150 \mathrm{mg}$ and placebo (Figure 2B). We observed disinhibition of SQSTM1, 
Figure 2 Longitudinal MicroRNA Expression
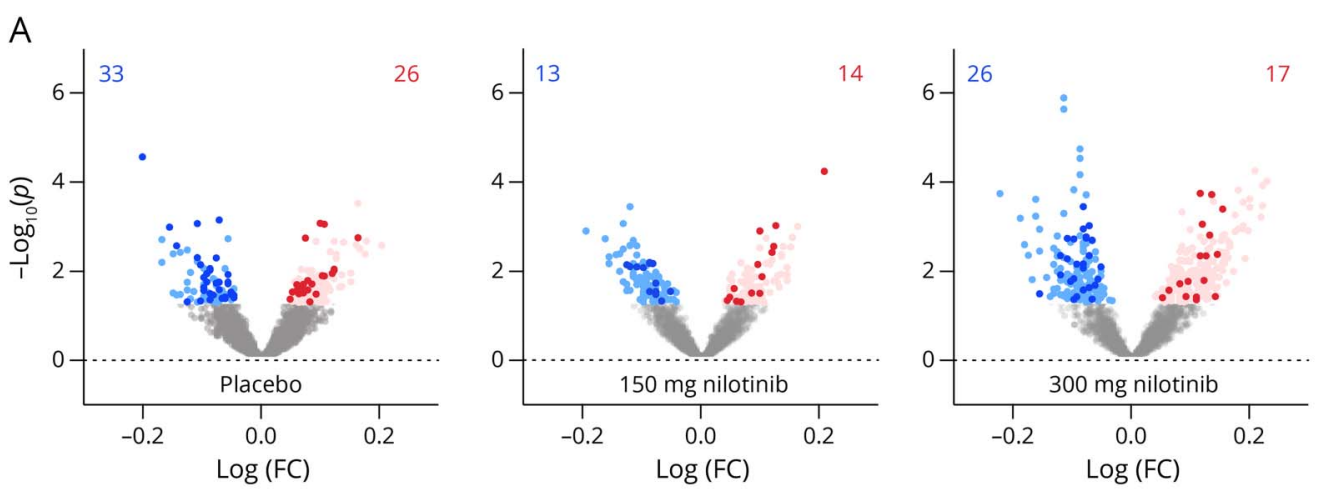

Expression Decrease Increase Wald test $(p<0.05)$ ME-ANOVA $(p<0.05)$

B

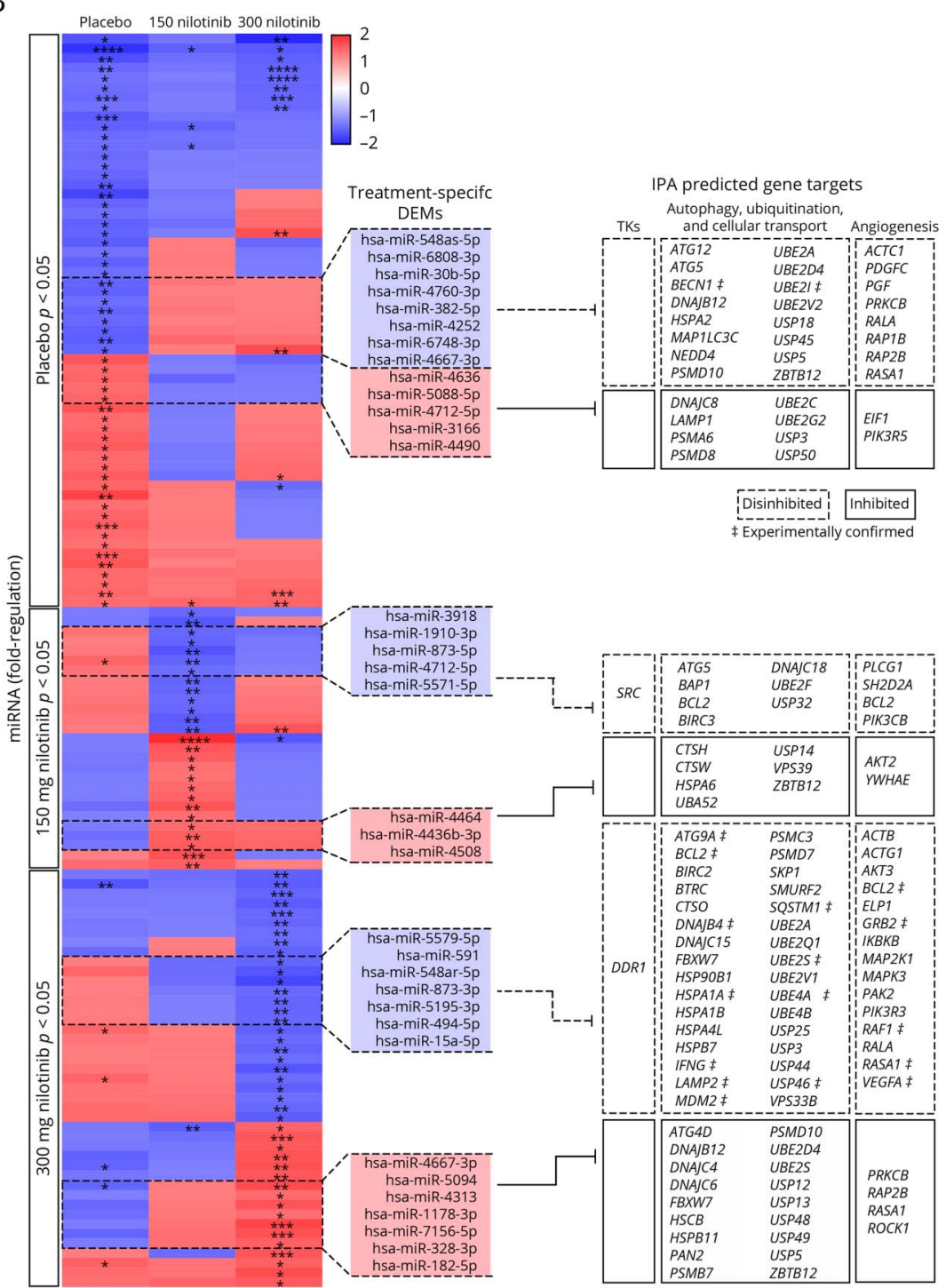

(A) Placebo, $150 \mathrm{mg}$ nilotinib, and $300 \mathrm{mg}$ nilotinib volcano plot of longitudinal miRNA expression in patients with Parkinson disease who underwent placebo treatment. Expression data are plotted as -log10 ( $p$-value) (y-axis) vs log (fold-change) (x-axis). Light colors indicate $p<0.05$ for the Wald test, and dark colors indicate that miRNA was also $p<0.05$, according to mixed-effects analysis of variance (ME-ANOVA) with the Kruskal-Wallis test. Blue indicates a decrease in expression, and red indicates an increase in expression. The number of miRNAs that meet both the Wald test and ME-ANOVA with the Kruskal-Wallis test $(p<$ $0.05)$ is shown in the top corners. (B) Heatmap of fold-regulation $\left(F R=F C^{-1}\right)$ for miRNAs $(p<0.05)$ that were in common between the Wald test and KruskalWallis test. ME-ANOVA $* p<0.05, * \star p<0.01, * \star \star p<0.001$, and $* \star \star \star x p<0.0001$. miRNA that displays an effect of treatment (expression directionality for nilotinib is opposite to placebo) is outlined by dotted line and shown to the right of the heatmap. Tyrosine kinase, autophagy, and angiogenesis predicted gene targets were determined via Ingenuity Pathway Analysis software (Qiagen) with confidence filtering to include only highly predicted gene targets. Gene targets with additional experimental confirmation are indicated by $¥$. Dashed lines indicate decreased miRNA expression leading to disinhibition of the gene targets, whereas bold line inhibition indicates increased inhibition of the gene targets. 
SMURF2, FBXW7, BTRC (hsa-miR-15a-5p), and SKP1 (hsamiR-5195), which mediate substrate recognition and recruitment for lysosomal degradation. These specific gene targets show that while initiation of lysosomal-autophagy is functioning, nilotinib, $300 \mathrm{mg}$, is able to facilitate the completion of these biological waste-control mechanisms. Nilotinib, $300 \mathrm{mg}$, significantly altered a number of ubiquitination genes and ubiquitin ligases, including NEDD4 (hsa-miR-30b-5p), UBEs (hsa-miR5a-5p, hsa-miR-4313, hsa-miR-4667-3p, and hsa-miR-548ar-5p), MDM (hsa-miR-5195), and PSMDs (hsa-miR-15a-5p and hsa-miR-4667-3p) as well as deubiquitination genes and ubiquitin-specific proteases (hsa-miR-5195-3p, hsa-miR-15a-5p, hsa-miR-182-5p, hsa-miR-4313, hsa-miR-7156-5p, hsa-miR-4667$3 p$, and hsa-miR-591), suggesting regulation of the ubiquitination/deubiquitination cycle, in agreement with previous reports. ${ }^{1,31}$ Alterations of autophagy-ubiquitination genes are also concurrent with significant changes of several heat shock proteins (hsa-miR-15a-5p, hsa-miR-5094, and hsa-miR-hsa-miR-873-3p) and vesicular transport genes (hsa-miR-15a-5p), suggesting facilitation of cellular transport ${ }^{1}$ and protein clearance.

\section{Alterations of miRNAs Targeting Genes That Regulate Collagen, Angiogenesis, and the BBB}

We expanded on our investigation of DEM gene regulation by performing unbiased gene ontology functional analysis using PANTHER classifications and pathway analysis using the g:Profiler online tool (Figure 3) using all gene targets determined by Ingenuity Pathway Analysis for each set of treatment-specific group subset as input. Remarkably, there was a significant enrichment of pathways associated with extracellular matrix (ECM) proteins (hsa-30b-5p and hsa-miR-7162-5p), various types of collagen (hsa-30b-5p and hsa-miR-7162-5p) and angiogenesis (hsa-30b-5p, hsa-miR-7162-5p, hsa-miR-4490, and hsa-miR$548 \mathrm{as}-5 \mathrm{p})$ in the placebo group, suggesting vascular changes (eFigure 2a, links.lww.com/NXG/A489 and Figure 3,A and B). Of interest, DDR1 is activated via autophosphorylation by collagen. ${ }^{5}$ Furthermore, the genes associated with nilotinib, $150 \mathrm{mg}$, group DEMs yielded no enriched pathways or GO terms compared with placebo, consistent with the low CSF concentration of nilotinib with this dose. However, in nilotinib, $300 \mathrm{mg}$, we observed enrichment of $\mathrm{BBB}$ maintenance pathways and regulation of transport across the BBB, specifically import of glucose (hsamiR-15a-5p, hsa-mIR-5195-3p, and hsa-miR-182-5p) (Figure 3, D-F) with reduced collagen signal, consistent with the CSF concentration of nilotinib that would inhibit DDR1. Additionally, we found enrichment of neurogenesis and gliogenesis (hsa-miR15a-5p, hsa-mIR-5195-3p, hsa-miR-182-5p, and hsa-miR-548ar$5 p$ ) (Figure 3D). There were also changes in several neuroinflammatory markers, including interleukin (hsa-miR15a-5p and hsa-mIR-5195-3p) signaling pathways, suggestive of molecular regulation of transport across the $\mathrm{BBB}$ (Figure 3C). These changes suggest that nilotinib, $300 \mathrm{mg}$, alters DDR1 signaling that may have an effect on the BBB, angiogenesis and vascular integrity. ${ }^{32,33}$ These changes in miRNA of ILs and other inflammatory factors and MMPs were seen in our previous CSF analysis of patients with PD and validated via RT-PCR quantification of relevant
miRNAs. Further analysis to validate other miRNA targets will be the scope of future studies.

\section{CSF miRNAs Associated With Angiogenesis Correlated With Decline of Motor and Nonmotor Symptoms}

We previously showed that nilotinib, 300 vs $150 \mathrm{mg}$, results in remarkable motor and nonmotor stability in medically optimized patients with PD over 27 months. ${ }^{21}$ In this study, we performed an exploratory analysis to show whether miRNA changes may affect clinical outcomes. Nilotinib-treated patients with PD were stable over 12 months, compared with placebo, using the sum of UPDRS II + III (Figure 4a, Table 2). In the placebo group, we observed an increase of 2.39 points in UPDRS II + III over 12 months (Figure 4, Table 2), whereas nilotinib, $150 \mathrm{mg}$ and $300 \mathrm{mg}$, remained stable over the same period. When placebo-treated patients were transitioned to nilotinib, 150 or $300 \mathrm{mg}$, for the second year, UPDRS II + III worsened 7.8 points in those treated with $150 \mathrm{mg}$ but improved -5.7 points in those treated with $300 \mathrm{mg}$ (Figure 4a, Table 2). Exploratory analysis showed that in the placebo group, 15 miRNAs significantly correlate with the increase in UPDRS II + III over 12 months (Figure $4 \mathrm{~b}$ ). Of interest, hsa-miR-133b is associated with receptor TK signaling, including vascular endothelial growth factor (VEGF)A-VEGFR2 signaling, indicative of angiogenesis (Figure 4c). We also performed analysis for Parkinson's Disease Questionnaire (PDQ)-39, in which we observed a similar trend to UPDRS II + III in scores among all treated groups (eFigure 3a, links.lww.com/NXG/A489). We observed 68 miRNAs that significantly correlate with the decline in PDQ-39 over 12 months (eFigure 3b, links.lww.com/NXG/ A489). These miRNAs (hsa-miR-135a-5p, hsa-miR-520e, hsamiR-134-5p, and hsa-miR-378d) are also associated with specific angiogenic pathways (proteoglycans, BDNF, TGF, and VEGF signaling) (eFigure 3c, links.lww.com/NXG/A489).

\section{Discussion}

This study primarily demonstrates alteration of CSF miRNAs, which regulate collagen, ECMs, and angiogenesis - as well as autophagy-lysosome pathways-over 12 months in patients with moderately severe $\mathrm{PD}$, suggesting impairment of these pathways in disease progression of these patients. Previous cross-sectional studies suggest that CSF biomarkers of angiogenesis $^{34}$ or autophagy ${ }^{4}$ are increased in PD. No longitudinal analysis was performed to demonstrate vascular impairment in Parkinson disease, thus this report represents novel findings that vascular defects may be present in the disease. We previously showed some changes in UPDRS over 12 months, ${ }^{15}$ but these changes were not statistically significant because of optimization of PD therapies in the placebo vs the nilotinib (150 $\mathrm{mg}$ and $300 \mathrm{mg}$ ) groups. Indeed, the present data show that UPDRS II + III worsened 2.39 points over 12 months in the placebo group, whereas nilotinib, $150 \mathrm{mg}$ and $300 \mathrm{mg}$, did not change. In addition, statistical significance between groups was not seen after 12 months of treatment primarily because 
Figure 3 Pathway and GO Functional Analysis Demonstrate Angiogenesis and BBB Breakdown Associated With Placebo and Inflammation and Autophagy Associated With 300 mg Nilotinib

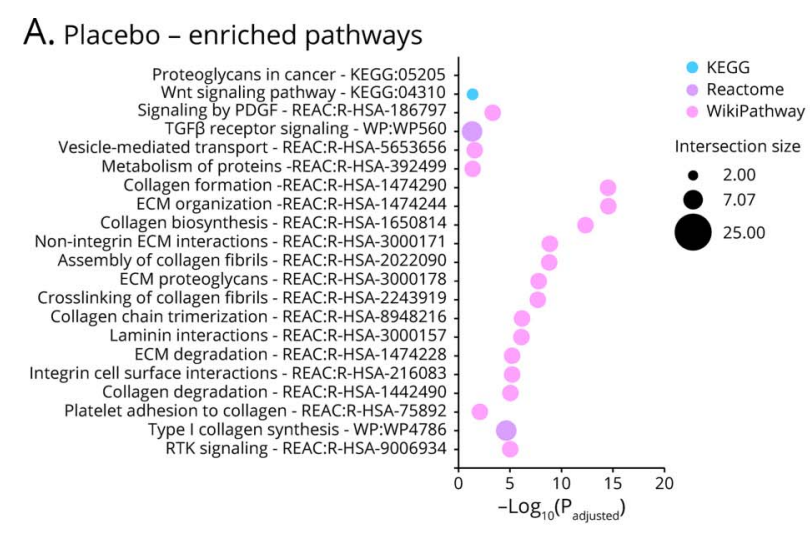

C. 300 mg nilotinib - enriched pathways

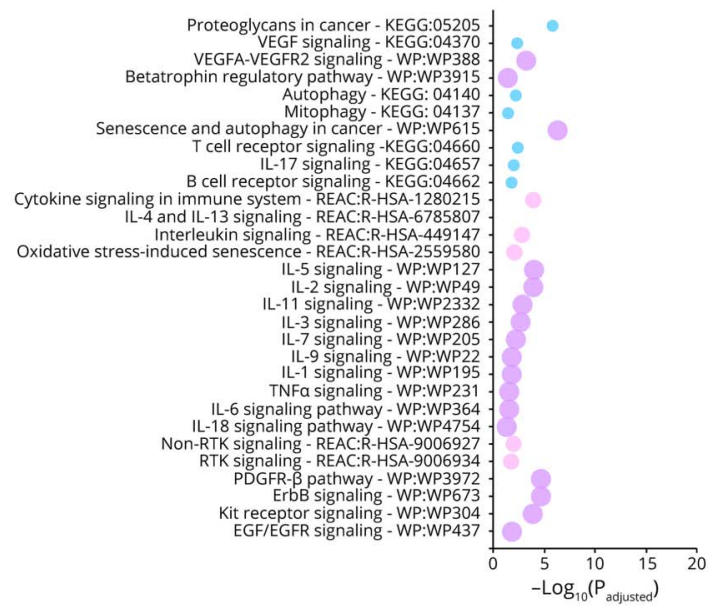

B. Placebo - enriched GO terms

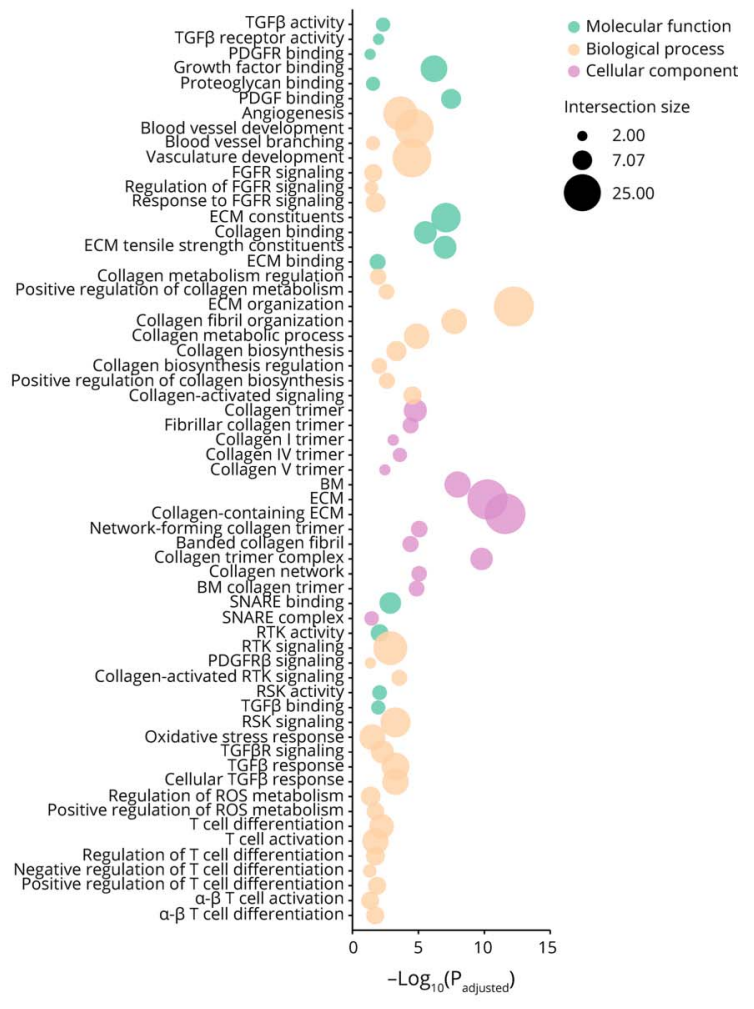

D. $300 \mathrm{mg}$ nilotinib - enriched GO terms

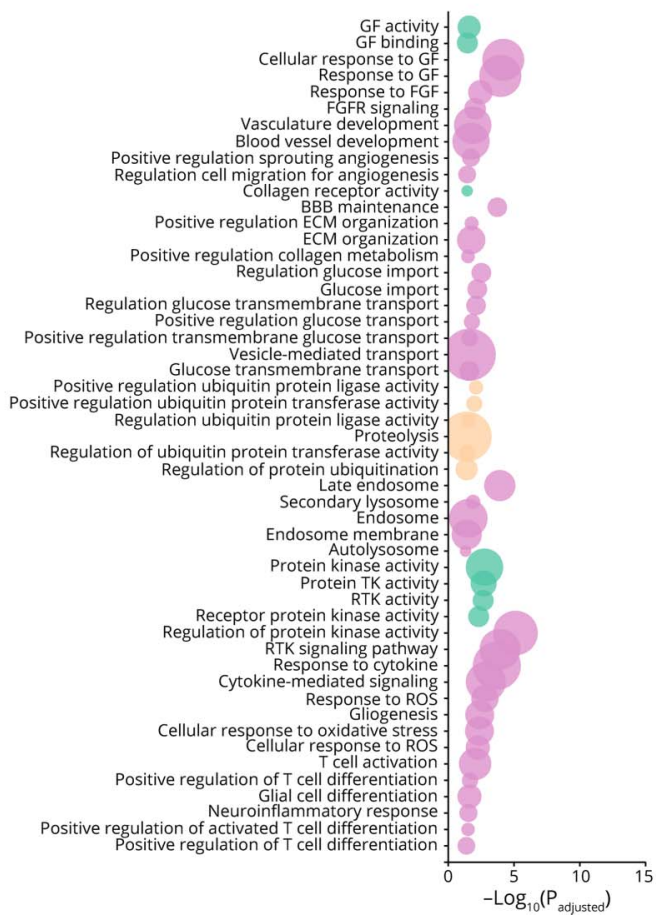

(A) KEGG (light blue), Reactome (pink), and WikiPathway (light purple) enrichment analysis for placebo differentially expressed miRNAs (DEMs). Enriched pathways were determined using the g:Profiler online tool with Benjamini-Hochberg multiple testing for significance $(p<0.05)$. (B) Gene ontology (GO) molecular function (green), biological process (orange), and cellular component (purple) enrichment analysis for placebo DEMs. (C) KEGG (light blue), Reactome (pink), and WikiPathway (light purple) enrichment analysis for placebo DEMs. Enriched pathways were determined using the g:Profiler online tool with Benjamini-Hochberg multiple testing for significance $(p<0.05)$. (D) GO molecular function (green), biological process (orange), and cellular component (purple) enrichment analysis for placebo DEMs. The bubble plot $x$-axis represents the - $\log 10\left(P_{\text {adj }}\right)$, and the size of the bubble corresponds to how many input genes intersect with the associated pathway or GO term. 


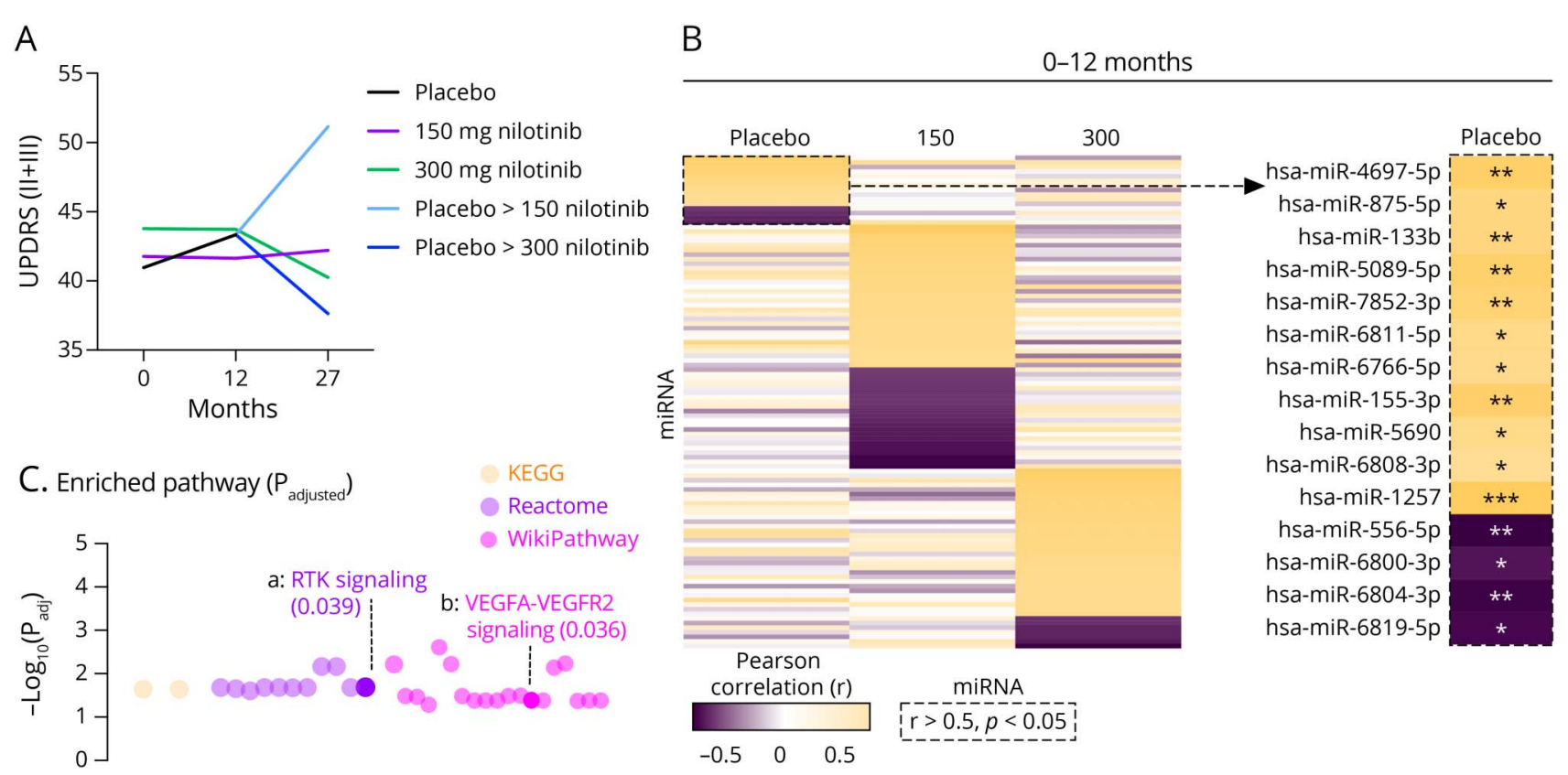

(A) The sum of Unified Parkinson's Disease Rating Score (UPDRS) II (motor) and III (activities of daily living) and at 0, 12, and 27 months \pm SD. The difference in scores is shown below in the table. (B) Heatmap of the correlation between 12-month and longitudinal changes in UPDRS II-III and longitudinal changes in miRNAs were computed using Spearman rank correlations. Placebo group miRNAs that highly correlate with UPDRS II + III ( $r>0.5, p<0.05)$ are outlined by a dotted line and are shown to the right. Twenty-seven-month correlation when placebo was transitioned to nilotinib, 150 or 300 mg, for the second year (right). (C) KEGG, Reactome, and WikiPathway enrichment analysis of gene regulation by miRNAs that highly correlate with UPDRS II + III in placebo.

the phase 2 study was statistically underpowered (by design) for clinical outcomes. ${ }^{15}$ However, long-term treatment with nilotinib 300 vs $150 \mathrm{mg}$ over 27 months showed significant clinical differences between the groups. ${ }^{21}$ When placebo was switched to nilotinib, 150 or $300 \mathrm{mg}$, for the second year, UPDRS II + III worsened 7.8 points in $150 \mathrm{mg}$, whereas it improved -5.7 points in nilotinib, $300 \mathrm{mg}$. The observed longitudinal changes of miRNAs and the correlation between miRNA changes and clinical outcomes suggest a diseasemodifying effect of nilotinib, $300 \mathrm{mg}$.

DDR1 is activated via binding to native forms of various collagens, ${ }^{5,35,36}$ and DDR1 activation in the CNS induces inflammation and BBB degradation. ${ }^{6}$ The current results show that several forms of collagens are expressed in PD, suggesting DDR1 activation, consistent with the upregulation of DDR 1 and 2 in postmortem brains of PD. ${ }^{4}$ Conversely, shRNA knockdown of DDR1 or DDR2 reduces the levels of neurotoxic proteins and prevents cell loss in vivo and in vitro. ${ }^{4}$ Furthermore, nilotinib, $300 \mathrm{mg}$, treatment for 1 year led to reduction of collagens and engagement of DDR1 miRNAs, suggesting changes of DDR1 activity. Collectively, these data suggest that DDR1 inhibition via nilotinib reverses the miRNA expression that led to vascular impairment in the CNS. Growth factors are required for the development of dopaminergic neurons, ${ }^{37}$ and they may rescue these neurons from toxicity. ${ }^{38}$ VEGF-expressing cells and blood vessels in the substantia nigra of parkinsonian monkeys were increased by chronic 1-methyl4-phenyl-1,2,3,6-tetrahydropyridine intoxication, ${ }^{39}$ suggesting that vascularization and growth factors may be upregulated to protect dopaminergic neurons. Indeed, changes in miRNAs that are associated with growth factors and angiogenesis in

Table 2 Longitudinal Changes in UPDRS II + III

\begin{tabular}{|c|c|c|c|c|c|c|}
\hline \multirow[b]{2}{*}{ Treatment } & \multicolumn{6}{|c|}{ UPDRS II + III longitudinal changes } \\
\hline & $\mathbf{O M}$ & $12 \mathrm{M}$ & $27 M$ & $\Delta 12-0 M$ & $\Delta 27-12 M$ & $\Delta$ 27-0M \\
\hline Placebo & $40.9 \pm 7.2$ & $43.3 \pm 12.2$ & - & 2.39 & - & - \\
\hline Placebo >150 nilotinib & - & - & $51.1 \pm 15$ & - & 7.8 & 10.19 \\
\hline Placebo >300 nilotinib & - & - & $37.6 \pm 9$ & - & -5.7 & -3.31 \\
\hline 150 nilotinib & $41.7 \pm 9.2$ & $41.6 \pm 11$ & $42.2 \pm 15.7$ & -0.14 & 0.57 & 0.43 \\
\hline 300 nilotinib & $43.7 \pm 8.3$ & $43.7 \pm 11$ & $40.2 \pm 11.1$ & -0.05 & -3.41 & -3.46 \\
\hline
\end{tabular}




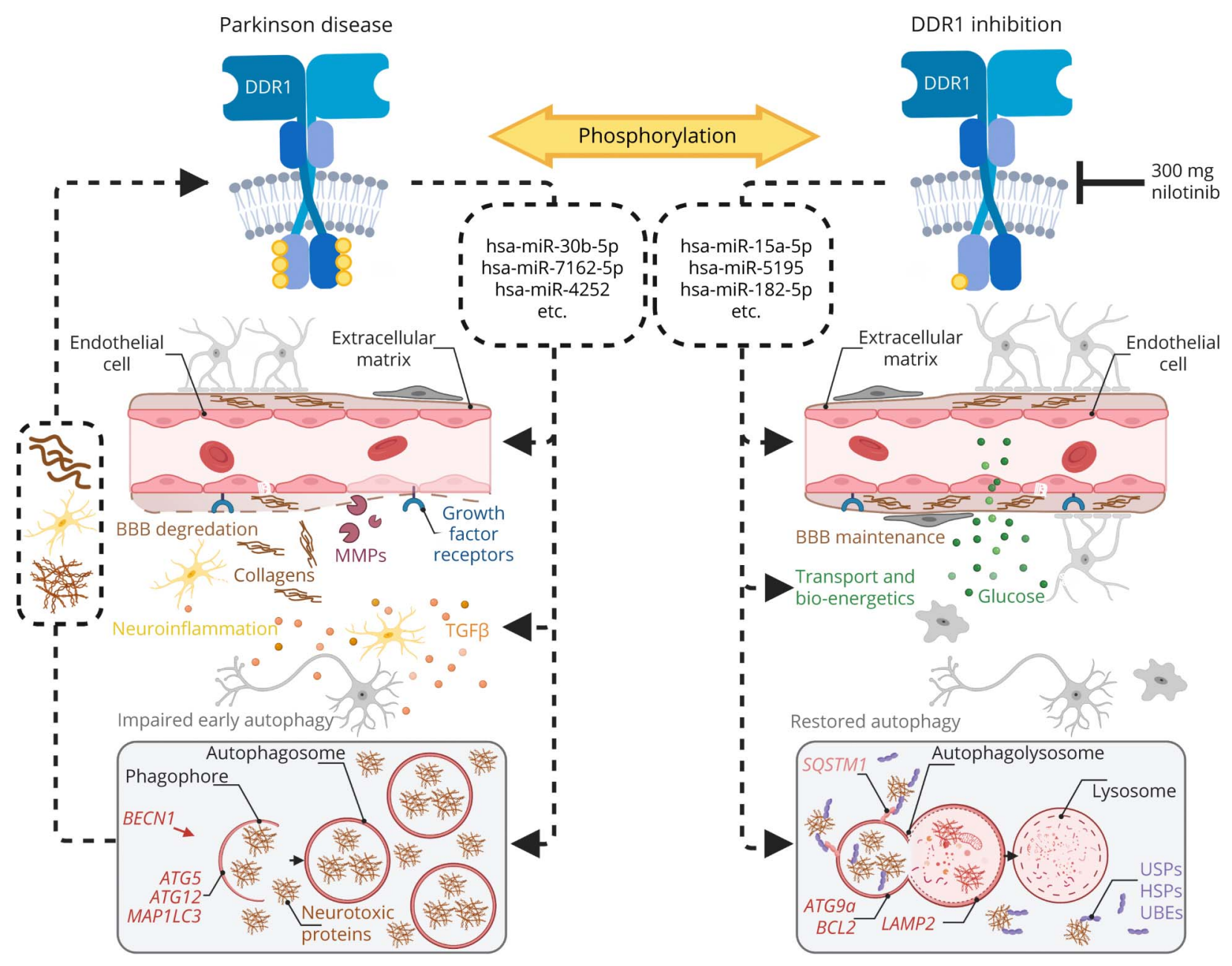

(A) Discoidin domain receptor 1 (DDR1) is overactivated, and several miRNAs, including hsa-miR-30b-5p, hsa-miR-7162-5p, and hsa-miR-4252, regulate several key Parkinson disease-associated pathologies, including blood-brain barrier (BBB) degradation, neuroinflammation, and impaired early autophagy. These pathologies may then promote DDR1 activity, creating a negative feedback loop. When DDR1 is inhibited, via nilotinib, $300 \mathrm{mg}$, we find that the miRNA differentially expressed miRNAs (DEMs) in placebo are reversed. In addition, we find that miRNAs, including hsa-miR-15a-5p, hsa-miR-5195, and hsa-miR182-5p, positively affect BBB maintenance, transport of glucose across the BBB, and bioenergetics, and restore autophagic flux. This schematic was created using BioRender.com.

nilotinib, $300 \mathrm{mg}$ vs placebo, are also associated with a significant increase in dopamine levels. ${ }^{15,16,21,40}$ Angiogenesis has not been extensively studied in PD, but postmortem evidence suggests that angiogenic vessels are present with markers of neuroinflammation in PD and other parkinsonism, suggesting infiltration of peripheral immune cells and inflammatory molecules to the parenchyma. ${ }^{41}$ Vascularization may modify the availability of nutrients, including glucose and alters neuroinflammatory signaling across the BBB. DDR1 attenuates inflammation in models of atherosclerosis. ${ }^{42}$ Taken together, our data suggest that vascularization may enhance transport across the $\mathrm{BBB}$ and contribute to a beneficial long-term immune response that would lead to potential clinical stabilization. ${ }^{21}$

Parkinson disease is associated with toxins, mitochondrial dysfunction, and impaired degradation of misfolded or abnormally aggregated proteins, but not vascular problems. Our data demonstrate that DDR1 inhibition may counteract alteration in the microvasculature that may be associated with impaired $\mathrm{BBB}$ and angiogenesis, leading to motor symptoms. ${ }^{34,43}$ Furthermore, clinical-pathologic studies indicate that the pathogenesis of age-related dementia is secondary to cerebrovascular disease, specifically atherosclerosis. ${ }^{44}$ Inhibition of DDR1 in nilotinib, $300 \mathrm{mg}$, was not only associated with motor and nonmotor stability but also did not result in cognitive deterioration in patients with PD with mild cognitive impairment. ${ }^{21}$ DDR1 inhibition may mitigate MMP effects on the BBB and neuroinflammation, while promoting angiogenesis and neurogenesis. ${ }^{45}$ There is evidence that DDR1 plays a direct role in angiogenesis, ${ }^{46}$ regulates the $\mathrm{BBB}^{47}$ via interaction with collagen, ${ }^{48}$ and involves epithelial cells as an ECM-sensor $^{49}$ (Figure 5) that controls inflammation. ${ }^{50}$ Targeting DDR1 is a feasible strategy to prevent $\mathrm{BBB}$ disruption and promotion of angiogenesis.

Examination of miRNAs that are known to regulate the TK targets of nilotinib (DDR1/2, Abelson) ${ }^{20}$ shows that only miRNAs in 
nilotinib, $300 \mathrm{mg}$, target DDR1. Nilotinib mechanism of action is thought to be at the level of the protein, but extended inhibition of DDR1 may result in a negative feedback loop that suppresses DDR1 gene expression. DDR1 activation regulates RhoA (ras homolog family member A) Ras, which increases atherosclerosis and the risk of cardiovascular events. ${ }^{32}$ DDR1 receptor signaling regulates the signaling pathways of cyclooxygenase-2 (COX-2), DARPP-32 (dopamine- and cAMP-regulated neuronal phosphoprotein), and BIK (Bcl-interacting killer); NF-kB (nuclear factor-kB); MEK (ERK activator kinase); and ERK (extracellular signal-regulated kinase). ${ }^{33}$ The expression of DDR1 in different types of cancer, including breast, renal cell carcinoma, non-smallcell lung carcinoma, esophageal, astrocytoma, prostate, hepatocellular carcinoma, and Hodgkin lymphoma, suggests a function in tumor progression, ${ }^{33}$ whereas DDR1 inhibition affects major biological pathways that can mitigate tumor progression.

DDR1 inhibition via nilotinib may result in activation of molecular pathways that mediate autophagy, ubiquitination, and mechanisms of cellular transport (Figure 5). Our previous data suggest that the increase in miRNAs that control autophagy and ubiquitination genes reduces expression of proteins that mediate ubiquitination and the endosomal and autophagy-lysosomal pathways, ${ }^{1}$ consistent with previous data that show impairment of autophagy in post-mortem Parkinson disease brains, ${ }^{2}$ and Nilotinib attenuates or reverses the level of miRNA expression, suggesting an increase in autophagy flux in agreement with animal data. ${ }^{9-13}$ Partial or complete knockdown, or pharmacologic inhibition of DDR1, attenuates neuroinflammation and improves CNS autophagy and vesicular transport. ${ }^{1,4,7-9}$

In conclusion, this longitudinal analysis of CSF miRNAs provides evidence of vascular impairment in the progression of $\mathrm{PD}$ and confirms the previous finding that autophagy and ubiquitination are impaired. DDR1 inhibition via nilotinib reverses miRNA changes that are indicative of vascular damage and autophagy dysfunction in PD.

This study has potential limitations including the short period (12 months) to observe greater changes in miRNAs that would underlie progression of clinical symptoms. The results of this study may be limited to the cohort of patients studied, and more studies are required to observe longitudinal changes of miRNAs in more disease stages of PD and further validation of changes of miRNA targets.

\section{Acknowledgment}

The authors thank the nurses and staff of the Clinical Research Unit at Georgetown University Medical Center, Center for Translational and Clinical Science, and all staff of the research pharmacy at MedStar Georgetown University Hospital and the Genomics and Epigenomics Shared Resource.

\section{Study Funding}

This study was supported by Georgetown University funds to C.M. and philanthropies through the Lasky and Barajas Family Fund and other private philanthropies. Nilotinib was provided to all participants by Novartis Pharmaceuticals Corporation. A.J. Fowler was supported through fellowships from the Georgetown-Howard Universities Center for Clinical and Translational Sciences' National Center for Advancing Translational Sciences' Clinical and Translational Science Award (TLR001431) and the National Institute for Neurological Disorders and Stroke (F31NS116938-01).

\section{Disclosure}

Y. Torres-Yaghi is an advisor. C. Moussa is an inventor on several US and international Georgetown University patents to use nilotinib and other tyrosine kinase inhibitors as a treatment for neurodegenerative diseases. C. Moussa's laboratory and Georgetown University previously received some income from licensing of the technology to Axovant Science, Georgetown University spun out the technology (April 2020) to a start-up company (KeifeRX LLC), from which C. Moussa receives consulting fees and Georgetown University. C. Moussa and F.L. Pagan receive equities. The remaining authors do not have any potential conflicts of interest to declare. Go to Neurology.org/NG for full disclosure.

\section{Publication History}

Received by Neurology: Genetics April 28, 2021. Accepted in final form August 27, 2021.

Appendix Authors

\begin{tabular}{|c|c|c|}
\hline Name & Location & Contribution \\
\hline $\begin{array}{l}\text { Alan Fowler, } \\
\text { MS }\end{array}$ & $\begin{array}{l}\text { Georgetown University, } \\
\text { Washington, DC }\end{array}$ & $\begin{array}{l}\text { Acquired and analyzed data } \\
\text { and drafted the manuscript }\end{array}$ \\
\hline $\begin{array}{l}\text { Jaeil Ahn, } \\
\text { PhD }\end{array}$ & $\begin{array}{l}\text { Georgetown University, } \\
\text { Washington, DC }\end{array}$ & $\begin{array}{l}\text { Acquired and analyzed data } \\
\text { and drafted the manuscript }\end{array}$ \\
\hline $\begin{array}{l}\text { Michealine } \\
\text { Hebron, MS }\end{array}$ & $\begin{array}{l}\text { Georgetown University, } \\
\text { Washington, DC }\end{array}$ & Acquired data \\
\hline $\begin{array}{l}\text { Timothy L. } \\
\text { Chiu, BS }\end{array}$ & $\begin{array}{l}\text { Georgetown University, } \\
\text { Washington, DC }\end{array}$ & Acquired data \\
\hline $\begin{array}{l}\text { Reem Ayoub, } \\
\text { BS }\end{array}$ & $\begin{array}{l}\text { Georgetown University, } \\
\text { Washington, DC }\end{array}$ & Acquired data \\
\hline $\begin{array}{l}\text { Sanjana } \\
\text { Mulki, MS }\end{array}$ & $\begin{array}{l}\text { Georgetown University, } \\
\text { Washington, DC }\end{array}$ & Acquired data \\
\hline $\begin{array}{l}\text { Habtom W. } \\
\text { Ressom, PhD }\end{array}$ & $\begin{array}{l}\text { Georgetown University, } \\
\text { Washington, DC }\end{array}$ & Acquired data \\
\hline $\begin{array}{l}\text { Yasar Torres- } \\
\text { Yaghi, MD }\end{array}$ & $\begin{array}{l}\text { Medstar Georgetown } \\
\text { University Hospital, } \\
\text { Washington, DC }\end{array}$ & Acquired data \\
\hline $\begin{array}{l}\text { Barbara } \\
\text { Wilmarth, } \\
\text { CRNP }\end{array}$ & $\begin{array}{l}\text { Georgetown University, } \\
\text { Washington, DC }\end{array}$ & Acquired data \\
\hline $\begin{array}{l}\text { Fernando L. } \\
\text { Pagan, MD }\end{array}$ & $\begin{array}{l}\text { Medstar Georgetown } \\
\text { University Hospital, } \\
\text { Washington, DC }\end{array}$ & Acquired data \\
\hline $\begin{array}{l}\text { Charbel } \\
\text { Moussa, } \\
\text { MBBS, PhD }\end{array}$ & $\begin{array}{l}\text { Georgetown University, } \\
\text { Washington, DC }\end{array}$ & $\begin{array}{l}\text { Conceived the study concept } \\
\text { and design and wrote and } \\
\text { finalized the manuscript }\end{array}$ \\
\hline
\end{tabular}




\section{References}

1. Fowler AJ, Hebron M, Balaraman K, et al. Discoidin Domain Receptor 1 is a therapeutic target for neurodegenerative diseases. Hum Mol Genet. 2020;29(17):2882-2898.

2. Lonskaya I, Hebron ML, Algarzae NK, Desforges N, Moussa CE. Decreased parkin solubility is associated with impairment of autophagy in the nigrostriatum of sporadic Parkinson's disease. Neuroscience. 2013;232:90-105.

3. Lonskaya I, Shekoyan AR, Hebron ML, Desforges N, Algarzae NK, Moussa CE. Diminished parkin solubility and co-localization with intraneuronal amyloid-beta are associated with autophagic defects in Alzheimer's disease. J Alzheimers Dis. 2013; 33(1):231-247.

4. Hebron M, Peyton M, Liu X, et al. Discoidin domain receptor inhibition reduces neuropathology and attenuates inflammation in neurodegeneration models. J Neuroimmunol. 2017;311(1):1-9.

5. Vogel W, Brakebusch C, Fassler R, Alves F, Ruggiero F, Pawson T. Discoidin domain receptor 1 is activated independently of beta(1) integrin. J Biol Chem. 2000;275(8): 5779-5784.

6. Zhu M, Xing D, Lu Z, et al. DDR1 may play a key role in destruction of the bloodbrain barrier after cerebral ischemia-reperfusion. Neurosci Res. 2015;96:14-19.

7. Fowler AJ, Hebron M, Missner AA, et al. Multikinase Abl/DDR/Src inhibition produces optimal effects for tyrosine kinase inhibition in neurodegeneration. Drugs $R$ D 2019;19(2):149-166.

8. Hebron ML, Lonskaya I, Moussa CE. Tyrosine kinase inhibition facilitates autophagic SNCA/alpha-synuclein clearance. Autophagy. 2013;9(8):1249-1250.

9. Hebron ML, Lonskaya I, Moussa CE. Nilotinib reverses loss of dopamine neurons and improves motor behavior via autophagic degradation of alpha-synuclein in Parkinson's disease models. Hum Mol Genet. 2013;22(16):3315-3328.

10. Lonskaya I, Desforges NM, Hebron ML, Moussa CE. Ubiquitination increases parkin activity to promote autophagic alpha-synuclein clearance. Plos One. 2013;8(12):e83914.

11. Lonskaya I, Hebron M, Desforges NM, Franjie A, Moussa CE. Tyrosine kinase inhibition increases functional parkin-Beclin-1 interaction and enhances amyloid clearance and cognitive performance. EMBO Mol Med. 2013;5(8):1247-1262.

12. Lonskaya I, Hebron M, Desforges NM, Schachter JB, Moussa CE. Nilotinib-induced autophagic changes increase endogenous parkin level and ubiquitination, leading to amyloid clearance. J Mol Med. 2014;92(4):373-386.

13. Hebron ML, Javidnia M, Moussa CE. Tau clearance improves astrocytic function and brain glutamate-glutamine cycle. J Neurol Sci. 2018;391:90-99.

14. Pagan F, Hebron M, Valadez EH, et al. Nilotinib effects in Parkinson's disease and dementia with Lewy bodies. J Parkinsons Dis. 2016;6(3):503-517.

15. Pagan FL, Hebron ML, Wilmarth B, et al. Nilotinib effects on safety, tolerability, and potential biomarkers in Parkinson disease: a phase 2 randomized clinical trial. JAMA Neurol. 2019;77(3):309-317.

16. Pagan FL, Hebron ML, Wilmarth B, et al. Pharmacokinetics and pharmacodynamics of a single dose Nilotinib in individuals with Parkinson's disease. Pharmacol Res Perspect. 2019;7(2):e00470.

17. Deremer DL, Ustun C, Natarajan K. Nilotinib: a second-generation tyrosine kinase inhibitor for the treatment of chronic myelogenous leukemia. Clin Ther. 2008;30(11): 1956-1975.

18. Jeitany M, Leroy C, Tosti P, et al. Inhibition of DDR1-BCR signalling by nilotinib as a new therapeutic strategy for metastatic colorectal cancer. EMBO Mol Med. 2018; 10(4):e7918.

19. Day E, Waters B, Spiegel K, et al. Inhibition of collagen-induced discoidin domain receptor 1 and 2 activation by imatinib, nilotinib and dasatinib. Eur J Pharmacol. 2008; 599(1-3):44-53.

20. Manley PW, Drueckes P, Fendrich G, et al. Extended kinase profile and properties of the protein kinase inhibitor nilotinib. Biochim Biophys Acta. 2010;1804(3):445-453.

21. Pagan FL, Wilmarth B, Torres-Yaghi Y, et al. Long-term safety and clinical effects of nilotinib in Parkinson's disease. Mov Disord. 2021;36(3):740-749.

22. Love MI, Huber W, Anders S. Moderated estimation of fold change and dispersion for RNA-seq data with DESeq2. Genome Biol. 2014;15(12):550.

23. Tarazona $S$, Furio-Tari $P$, Turra $D$, et al. Data quality aware analysis of differential expression in RNA-seq with NOISeq R/Bioc package. Nucleic Acids Res. 2015;43(21):e140.

24. Mi H, Muruganujan A, Huang X, et al. Protocol Update for large-scale genome and gene function analysis with the PANTHER classification system (v.14.0). Nat Protoc. 2019;14(3):703-721.
25. Orr M, Liu P. Sample size estimation while controlling false discovery rate for microarray experiments using ssize.fdr package. $R$ J. 2009;1:47-53.

26. Chou CH, Shrestha S, Yang CD, et al. miRTarBase update 2018: a resource for experimentally validated microRNA-target interactions. Nucleic Acids Res. 2018; 46(D1):D296-D302.

27. Klionsky DJ, Abdelmohsen K, Abe A, et al. Guidelines for the use and interpretation of assays for monitoring autophagy (3rd edition). Autophagy. 2016;12(1):1-222.

28. Fowler AJ, Moussa CE. Activating autophagy as a therapeutic strategy for Parkinson's disease. CNS Drugs. 2018;32(1):1-11.

29. Eskelinen EL. Roles of LAMP-1 and LAMP-2 in lysosome biogenesis and autophagy. Mol Aspects Med. 2006;27(5-6):495-502.

30. Saftig P, Beertsen W, Eskelinen EL. LAMP-2: a control step for phagosome and autophagosome maturation. Autophagy. 2008;4(4):510-512.

31. Liu X, Hebron M, Shi W, Lonskaya I, Moussa CE. Ubiquitin specific protease-13 independently regulates parkin ubiquitination and alpha-synuclein clearance in alphasynucleinopathies. Hum Mol Genet. 2019;28(4):548-560.

32. Ngai D, Lino M, Rothenberg KE, Simmons CA, Fernandez-Gonzalez R, Bendeck MP. DDR1 (discoidin domain receptor-1)-RhoA (ras homolog family member A) axis senses matrix stiffness to promote vascular calcification. Arterioscler Thromb Vasc Biol. 2020;40(7):1763-1776.

33. Jing H, Song J, Zheng J. Discoidin domain receptor 1: new star in cancer-targeted therapy and its complex role in breast carcinoma. Oncol Lett. 2018;15(3):3403-3408.

34. Munoz DG, Woulfe JM. Angiogenesis: a new paradigm for Parkinson disease with practical and pathogenic implications. Neurology. 2015;85(21):1826-1827.

35. Vogel W, Gish GD, Alves F, Pawson T. The discoidin domain receptor tyrosine kinases are activated by collagen. Mol Cel. 1997;1(1):13-23.

36. Shrivastava A, Radziejewski C, Campbell E, et al. An orphan receptor tyrosine kinase family whose members serve as nonintegrin collagen receptors. Mol Cel. 1997;1(1):25-34.

37. Ratzka A, Baron O, Grothe C. FGF-2 deficiency does not influence FGF ligand and receptor expression during development of the nigrostriatal system. PLoS One. 2011; 6(8): 23564 .

38. Timmer M, Cesnulevicius K, Winkler C, et al. Fibroblast growth factor (FGF)-2 and FGF receptor 3 are required for the development of the substantia nigra, and FGF-2 plays a crucial role for the rescue of dopaminergic neurons after 6-hydroxydopamine lesion. J Neurosci. 2007;27(3):459-471.

39. Barcia C, Bautista V, Sanchez-Bahillo A, et al. Changes in vascularization in substantia nigra pars compacta of monkeys rendered parkinsonian. J Neural Transm (Vienna). 2005;112(9):1237-1248.

40. Turner RS, Hebron ML, Lawler A, et al. Nilotinib effects on safety, tolerability, and biomarkers in Alzheimer's disease. Ann Neurol. 2020;88(1):183-194.

41. Desai Bradaric B, Patel A, Schneider JA, Carvey PM, Hendey B. Evidence for angiogenesis in Parkinson's disease, incidental Lewy body disease, and progressive supranuclear palsy. J Neural Transm (Vienna). 2012;119(1):59-71.

42. Franco C, Hou G, Ahmad PJ, et al. Discoidin domain receptor 1 (ddr1) deletion decreases atherosclerosis by accelerating matrix accumulation and reducing inflammation in low-density lipoprotein receptor-deficient mice. Circ Res. 2008; 102(10):1202-1211.

43. Janelidze S, Lindqvist D, Francardo V, et al. Increased CSF biomarkers of angiogenesis in Parkinson disease. Neurology. 2015;85(21):1834-1842.

44. Raz L, Knoefel J, Bhaskar K. The neuropathology and cerebrovascular mechanisms of dementia. J Cereb Blood Flow Metab. 2016;36(1):172-186.

45. Rosenberg GA. Matrix metalloproteinases and their multiple roles in neurodegenerative diseases. Lancet Neurol. 2009;8(2):205-216.

46. Oh S, Seo M, Choi JS, Joo CK, Lee SK. MiR-199a/b-5p inhibits lymphangiogenesis by targeting discoidin domain receptor 1 in corneal injury. Mol Cell. 2018;41(2):93-102.

47. Stamatovic SM, Keep RF, Andjelkovic AV. Brain endothelial cell-cell junctions: how to "open" the blood brain barrier. Curr Neuropharmacol. 2008;6(3):179-192.

48. Leitinger B, Saltel F. Discoidin domain receptors: multitaskers for physiological and pathological processes. Cell Adh Migr. 2018;12(4):398-399.

49. Roberts ME, Magowan L, Hall IP, Johnson SR. Discoidin domain receptor 1 regulates bronchial epithelial repair and matrix metalloproteinase production. Eur Respir J. 2011;37(6):1482-1493.

50. Dorison A, Dussaule JC, Chatziantoniou C. The role of discoidin domain receptor 1 in inflammation, fibrosis and renal disease. Nephron. 2017;137(3):212-220. 


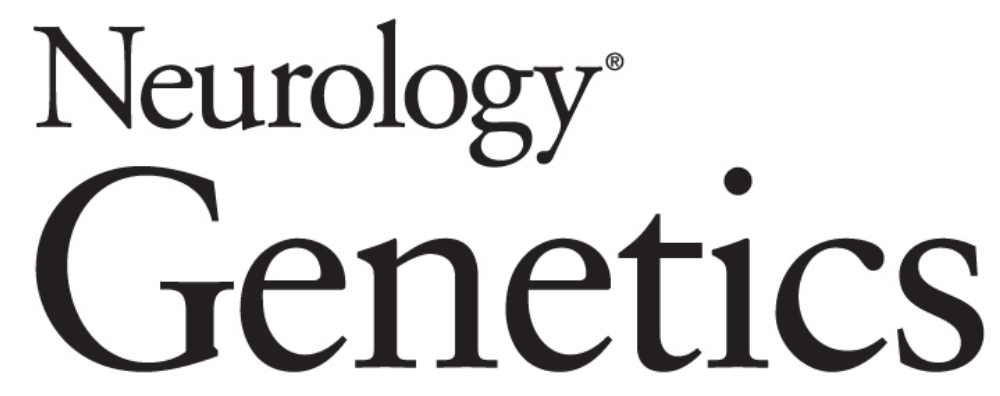

\section{CSF MicroRNAs Reveal Impairment of Angiogenesis and Autophagy in Parkinson Disease}

Alan J. Fowler, Jaeil Ahn, Michaeline Hebron, et al. Neurol Genet 2021;7;

DOI 10.1212/NXG.0000000000000633

This information is current as of November 12, 2021

\section{Updated Information \&} Services

References

Subspecialty Collections

Permissions \& Licensing

Reprints including high resolution figures, can be found at: http://ng.neurology.org/content/7/6/e633.full.html

This article cites 50 articles, 6 of which you can access for free at: http://ng.neurology.org/content/7/6/e633.full.html\#\#ref-list-1

This article, along with others on similar topics, appears in the following collection(s):

\section{Parkinson's disease/Parkinsonism}

http://ng.neurology.org//cgi/collection/parkinsons_disease_parkinsonis $\mathrm{m}$

Information about reproducing this article in parts (figures,tables) or in its entirety can be found online at:

http://ng.neurology.org/misc/about.xhtml\#permissions

Information about ordering reprints can be found online: http://ng.neurology.org/misc/addir.xhtml\#reprintsus

Neurol Genet is an official journal of the American Academy of Neurology. Published since April 2015, it is an open-access, online-only, continuous publication journal. Copyright Copyright ( 2021 The Author(s). Published by Wolters Kluwer Health, Inc. on behalf of the American Academy of Neurology.. All rights reserved. Online ISSN: 2376-7839.

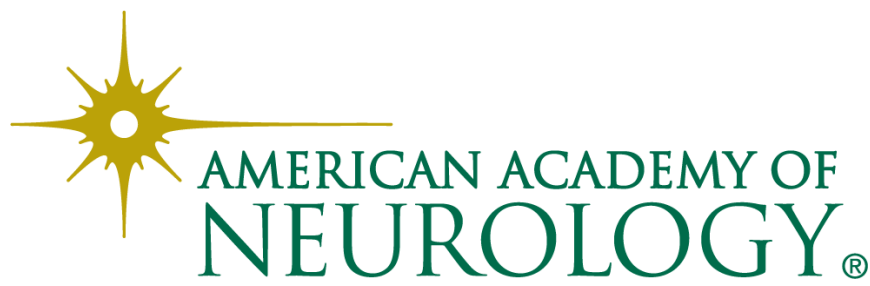

